\title{
Three-dimensional strong localization of matter waves by scattering from atoms in a lattice with a confinement-induced resonance
}

\author{
Pietro Massignan \\ Niels Bohr Institutet, Universitetsparken 5, DK-2100 Copenhagen Ø, Denmark, and \\ Laboratoire Kastler Brossel, École Normale Supérieure, 24 rue Lhomond, 75005 Paris, France. \\ Yvan Castin \\ Laboratoire Kastler Brossel, École Normale Supérieure, 24 rue Lhomond, 75005 Paris, France.
}

(Dated: November 7, 2018)

\begin{abstract}
The possibility of using ultracold atoms to observe strong localization of matter waves is now the subject of a great interest, as undesirable decoherence and interactions can be made negligible in these systems. It was proposed that a static disordered potential can be realized by trapping atoms of a given species in randomly chosen sites of a deep 3D optical lattice with no multiple occupation. We analyze in detail the prospects of this scheme for observing localized states in 3D for a matter wave of a different atomic species that interacts with the trapped particles and that is sufficiently far detuned from the optical lattice to be insensitive to it. We demonstrate that at low energy a large number of 3D strongly localized states can be produced for the matter wave, if the effective scattering length describing the interaction of the matter wave with a trapped atom is of the order of the mean distance between the trapped particles. Such high values of the effective scattering length can be obtained by using a Feshbach resonance to adjust the free space inter-species scattering length and by taking advantage of confinement-induced resonances induced by the trapping of the scatterers in the lattice.
\end{abstract}

PACS numbers:

\section{INTRODUCTION}

The recent advances in the manipulation of ultracold gases have made it possible to employ these systems to accurately simulate several non-trivial problems in condensed matter physics. As examples, we may mention the exploration of the BCS to BEC crossover [1] and the superfluid to Mott-insulator transition [2]. Disorder plays an important role in the theory of solid state, affecting in a substantial way the transport properties of various systems. Special attention has in the past been dedicated to studies of light propagation in strongly-scattering powders and electron transport in the presence of impurities $[3,[4,[5]$.

It looks therefore interesting to introduce a controlled disorder in the experiments with ultracold atoms, in order to provide a closer modeling of realistic systems of condensed matter physics. It was indeed predicted that atomic gases stored in optical lattices would be good candidates to experimentally observe the effect of a disordered or quasi-periodic potential on an interacting Bose gas or on interacting Fermi-Bose mixture 6, 7]. First experimental results along this research line have been recently reported, i.e. the observation of a Bose glass in a quasi-periodic potential [8] and the study of spatial coherence properties of an interacting Bose gas trapped in a lattice in presence of a disordered ensemble of fermionic atoms [9].

In this paper, we consider a variant of this line of research, that is the possibility of looking for genuine localized states of a non-interacting matter wave exposed to static disorder in continuous space. Localized states are stationary states with a square integrable wave function at an energy where the classical motion is not bounded spatially. In a paper that dates back to the early years of quantum mechanics, von Neumann and Wigner 10 showed that the Schrödinger equation can admit square integrable eigenstates embedded in the continuum of states with energy higher than the maximum of the potential. After the work of Anderson [11], it is expected that disordered potentials can generically lead in $3 \mathrm{D}$ to a quantum phase transition, a macroscopic number of localized states being present at low energy. Such a phase transition in $3 \mathrm{D}$ is not straightforward to observe, as it is sensitive to decoherence and wave absorption effects, and requires a mean free path of the wave $l$ smaller than its wave length $\lambda$, as stated by the Ioffe-Regel criterion

$$
\lambda \gtrsim l
$$

The study of localization of light is a well developed experimental subject: strong localization of light has been reported in semi-conductor powders 12, and weak localization effects of light in a gas of cold atoms are the subject of an intensive experimental study [13]. On the contrary, for matter waves, no direct evidence of localization was obtained in 3D. Matter waves made of ultracold atoms are good candidates in this respect, due to their weak coupling to the environment and to the possibility of tuning their interactions with a Feshbach resonance [14]. An open problem is however to know if strong enough disorder can be introduced in these gases to lead to reasonably short localization lengths in $3 \mathrm{D}$.

A natural way to produce a disordered potential in atomic gases is to use the speckle pattern of a laser beam 


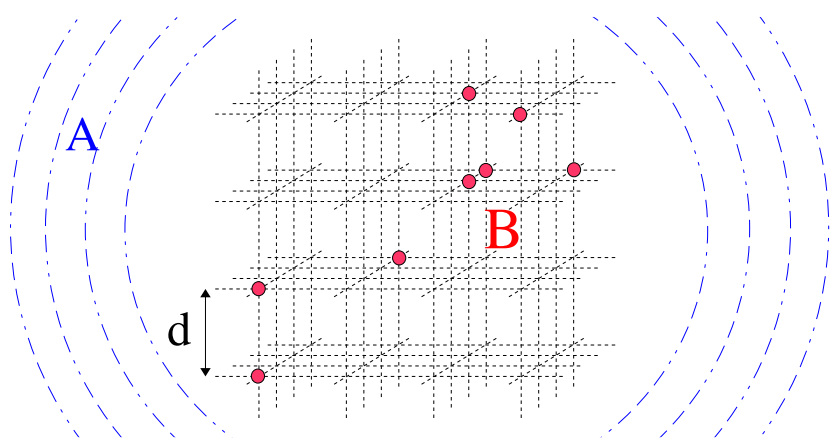

FIG. 1: (Color online) Sketch of the model of disorder considered here: a matter wave (A, blue) scatters on randomly distributed B particles (red), each occupying the vibrational ground state of a node in a 3D optical lattice (here the average occupancy $p$ is 0.1 ). The lattice does not act on the matter wave $\mathrm{A}$.

15. Many experiments on Bose-Einstein condensates in $1 \mathrm{D}$ random optical potentials have very recently been reported 16, 17, 18, 19], and they provide evidence for disorder-related effects such as fragmentation of the condensate, suppression of diffusion, frequency shifts and damping of collective oscillations. Theoretically, these effects were discussed in [20]. Genuine strong localization in $1 \mathrm{D}$, in the non-interacting regime, has not been reported yet in these experiments, and the implementation of the disordered optical potential in $3 \mathrm{D}$ remains to be done. Also the theoretical analysis of matter wave localization in a speckle pattern, recently performed in 2D [21], has not been done in a detailed way in the 3D strong localization regime.

An alternative method to realize a disordered potential was proposed in 22]: a matter wave, made of atoms of a species $A$, scatters off a set of atoms of another species $B$ that are trapped in randomly chosen sites of a deep optical lattice with no multiply occupied sites (see Fig. \). As we will choose the lattice to be very far detuned for the species $A$, the matter wave moves unaffected through the optical lattice, and interacts only with the $B$ atoms: this excludes classical localization effects in local potential minima. The disorder can be made very strong, since (i) the correlation length of the disorder can be as small as $0.5 \mu \mathrm{m}$ (the spatial period of the optical lattice), and (ii) the scattering cross section of the matter wave off a single $B$ atom can be made as high as allowed by quantum mechanics (the so-called unitary limit) by use of a $A-B$ interspecies Feshbach resonance, making it possible to dramatically reduce the mean free path of the matter wave. Furthermore, as we shall take advantage of, this model allows a straightforward exact numerical analysis even in 3D, when $B$ atoms are modeled as fixed point-like scatterers, as is known for light waves 23, 24].

It is the $3 \mathrm{D}$ version of this scheme that we analyze in this paper. After the detailed presentation of our model and its practical implementation given in Sec. III we show numerically in Sec. III that it leads to the appearance of a large number of localized states for a range of parameters accessible in present experiments, provided that the effective coupling between the matter wave and a single scatterer is tuned to a value of the order of the mean scatterer separation. Section IV is dedicated to the quantitative description of the scattering between the matter wave and a single trapped scatterer, and to the discussion of the confinement-induced resonances thereby arising: we show indeed that large enough effective coupling constants can be obtained. Our conclusions are presented in Sec. $\mathbb{\nabla}$ including a discussion of a possible strategy of observation of strong localization.

\section{OUR MODEL}

\section{A. The disordered potential}

The scatterers are a set of identical particles, whose chemical species and quantum numbers will be indexed by the letter $B$, randomly occupying (with filling factor $p<1$ ) the sites of a 3D cubic optical lattice. The potential seen by the $B$ atoms is produced by a superposition of three laser standing waves of common intensity and mutually orthogonal linear polarizations along the $x, y$ and $z$ axes,

$$
V^{B}(\mathbf{r})=V_{0}^{B}\left[\sin ^{2}\left(k_{L} x\right)+\sin ^{2}\left(k_{L} y\right)+\sin ^{2}\left(k_{L} z\right)\right],
$$

where $V_{0}^{B}>0$ is the modulation depth of the lattice and $k_{L}=2 \pi / \lambda_{L}$ is the laser wavenumber. We shall denote the lattice spatial period along each axis by $d=\lambda_{L} / 2=\pi / k_{L}$. Multiple occupation of a lattice well is assumed to be absent, by use of polarized fermions, or by creation of vacancies in a unit occupancy Mott phase state [2], or simply by choosing $p$ sufficiently small to make it statistically irrelevant.

We choose the lattice depth $V_{0}^{B}$ to be much larger than the recoil energy $E_{r}^{B}=\hbar^{2} k_{L}^{2} / 2 m_{B}$ of the $B$ atoms so that the tunneling time of $B$ atoms from one lattice site to another $\left(t_{\text {tunnel }} \approx 1.6 \times 10^{4} \hbar / E_{r}^{B}\right.$ for $\left.V_{0}^{B}=50 E_{r}^{B}\right)$ is negligible over the duration of the experiment and the disordered spatial pattern of $B$ atoms is static [27].

We also require that each $B$ atom is prepared in the vibrational ground state of the local lattice micro-trap, which can be achieved in practice by raising adiabatically the optical lattice on top of a condensate cloud of atoms $B$ [2], or by applying Raman laser cooling sideband techniques [25, 26] to an optical molasses. This condition is crucial to ensure that each $A-B$ scattering event is elastic when the $A$ atoms have sufficiently low energy: indeed, energy conservation guarantees that the $B$ atom is left in the vibrational ground state after scattering with a $A$ atom of momentum $k$ if

$$
\frac{\hbar^{2} k^{2}}{2 m_{A}} \ll \hbar \omega=2\left(V_{0}^{B} E_{r}^{B}\right)^{1 / 2}
$$


where $\omega$ is the oscillation frequency of a $B$ atom in a micro-trap.

A last point is to ensure that spontaneous emission processes are negligible for the $B$ atoms. In order to achieve large values of $V_{0}^{B}$ with negligible heating of the trapped scatterers, we require the lattice to be blue-detuned with respect to the strongest transition of the $B$ atoms (in blue-detuned lattices, particles are trapped in the minima of intensity of the stationary light field). Including the Lamb-Dicke type reduction factor coming from the trapping of $B$ atoms close to the nodes of the laser field, one gets the fluorescence rate

$$
\Gamma_{\text {fluo }}^{B}=\Gamma_{B} \frac{V_{0}^{B}}{\omega_{L}-\omega_{B}} \frac{3 k_{L}^{2}}{2 m_{B} \omega}
$$

where $\omega_{L}-\omega_{B}$ is the atom-laser detuning and $\Gamma_{B}$ is the spontaneous emission rate of $B$ atoms.

For the bosonic ${ }^{87} \mathrm{Rb}$ isotope of rubidium $\left(\lambda_{B, D 2}=\right.$ $\left.780 \mathrm{~nm}, \lambda_{B, D 1}=794.8 \mathrm{~nm}\right)$ and an optical lattice tuned at $\lambda_{L}=779 \mathrm{~nm}$, only at $1 \mathrm{~nm}$ to the blue of the strongest rubidium transition at $780 \mathrm{~nm}$, at the required lattice intensity $V_{0}^{B}=50 E_{r}^{B}$ the tunneling time is $t_{\text {tunnel }} \approx 0.7 \mathrm{~s}[27]$ and the fluorescence rate is $\Gamma_{\text {fluo }}^{B} \sim 10^{-4} E_{r}^{B} / \hbar \sim 3 \mathrm{~s}^{-1}$, allowing experimental times up to $300 \mathrm{~ms}$. The same calculation, taking for $B$ the fermionic isotope ${ }^{40} \mathrm{~K}$ of potas$\operatorname{sium}\left(\lambda_{B, D 2}=766.5 \mathrm{~nm}, \lambda_{B, D 1}=769.9 \mathrm{~nm}\right)$ and an optical wavelength $\lambda=765.5 \mathrm{~nm}$ leads to $t_{\text {tunnel }} \approx 0.32 \mathrm{~s}$ and $\Gamma_{\text {fluo }}^{B} \sim 5 \mathrm{~s}^{-1}$.

\section{B. Model Hamiltonian for the matter wave and its limitations}

The matter wave to be strongly-localized is made of atoms of another species, that we will label by $A$. We shall ignore interaction effects among these $A$ atoms. One way to fulfill this condition in a real experiment would be to take spin polarized fermionic atoms: $s$-wave interactions are prohibited by the exclusion principle and $p$-wave interactions are very weak at low energies in the absence of a $p$-wave resonance.

The $A$ atoms experience interactions with the trapped species $B$. At low incoming kinetic energy of a $A$ particle, we model these interactions by static contact potentials, corresponding to infinitely-massive point-like scatterers, each located at the center of a micro-well occupied by a $B$ atom:

$$
\mathcal{V}=\frac{2 \pi \hbar^{2} a_{\mathrm{eff}}}{m_{A}} \sum_{j=1}^{N} \delta\left(\mathbf{r}_{A}-\mathbf{r}_{j}\right) \partial_{\left|\mathbf{r}_{A}-\mathbf{r}_{j}\right|}\left(\left|\mathbf{r}_{A}-\mathbf{r}_{j}\right| \ldots\right)
$$

where the sum is taken over the $N$ scatterers. The effective scattering length $a_{\text {eff }}$ of a $A$ atom on a trapped $B$ atom, when expressed in units of the harmonic-oscillator length $a_{\mathrm{ho}}=\sqrt{\hbar / m_{B} \omega}$, is a function of the dimensionless ratios $m_{B} / m_{A}$ and $a / a_{\mathrm{ho}}, a$ being the $A-B$ scattering length in free space. The value of $a_{\text {eff }}$ and the validity condition of our model potential, Eq. (5), will be given in Sec. IIV

The $A$ atoms also experience the optical lattice potential, with the same spatial dependence as in Eq. (2) but with a different modulation amplitude $V_{0}^{A}$. We require the optical lattice to be much closer to resonance with $B$ atoms than with $A$ atoms, $\left|\omega_{L}-\omega_{A}\right| \gg\left|\omega_{L}-\omega_{B}\right|$, such that $V_{0}^{A}$ will be much smaller than $V_{0}^{B}$. In particular, we impose that

$$
\left|V_{0}^{A}\right| \ll E_{r}^{A}=\frac{\hbar^{2} k_{L}^{2}}{2 m_{A}}
$$

so that, in the absence of $B$ atoms, the $A$ atoms can be safely considered as free. In this respect, a particularly promising combination is given by fermionic ${ }^{6} \mathrm{Li}$ for the species $A\left(\lambda_{A}=671 \mathrm{~nm}\right)$ and ${ }^{87} \mathrm{Rb}$ for the species $B$ : taking $\lambda_{L}=779 \mathrm{~nm}$ and a laser intensity such that $V_{0}^{B}=$ $50 E_{r}^{B}$, one finds $V_{0}^{A}=-0.04 E_{r}^{A}$. If one takes for $B$ the fermionic ${ }^{40} \mathrm{~K}$ with $\lambda_{L}=765.5 \mathrm{~nm}$, one finds $V_{0}^{A}=$ $-0.09 E_{r}^{A}[28]$.

We shall therefore neglect the effect of the optical lattice on the $A$ atoms and take as a model Hamiltonian for the matter wave:

$$
\mathcal{H}=\mathcal{H}_{0}+\mathcal{V} \quad \text { with } \quad \mathcal{H}_{0}=-\frac{\hbar^{2}}{2 m_{A}} \Delta_{\mathbf{r}_{A}} .
$$

We note in passing that in the original Anderson model the $A$ particles were instead assumed to be in the tightbinding regime, so that the strong localization reported in this paper is not stricto sensu Anderson localization.

To end this section, we briefly discuss two effects not included in our model Hamiltonian that may impose limitations in a real experiment. As we shall see, the production of localized states with a short localization length (of the order of the lattice spacing $d$ ) requires the use of a large and positive value of $a_{\text {eff }} \sim d$, obtained by a $A-B$ Feshbach resonance. As a consequence, the matter wave $A$ has a weakly bound state with a trapped atom $B$, of spatial extension $\sim a_{\text {eff }}$. A first undesired effect is therefore the formation of such $A-B$ dimers.

A first stage that may lead to a dimer production is during the Feshbach ramp of $a_{\text {eff }}$ from $\sim 0$ to $\sim d$. This may be avoided by using a ramping time longer than the inverse of the dimer binding frequency, $2 m_{A} a_{\text {eff }}^{2} / \hbar \sim 30 \mu \mathrm{s}$ for our previous example with lithium and rubidium. Once $a_{\text {eff }}$ is set to $\sim d$, one may fear that three-body collisions $A+A+B$ lead to the formation of a dimer. For our model Hamiltonian Eq. (17) the $A$ particles are an ideal gas and the dimer formation does not happen: the trapped $B$ particle, being replaced by a fixed scatterer with no degree of freedom, cannot mediate a $A-A$ interaction. In the opposite limit where the $B$ scatterer is supposed to move freely, the rate of dimer formation per $B$ atom is $\gamma_{\operatorname{dim}}=C_{\operatorname{dim}}\left(k_{F} d\right)^{8} E_{r}^{A} / \hbar$, where we used Eqs. $(11,12)$ of [29], taking a dimer binding energy $\epsilon=\hbar^{2} / 2 \mu a_{\mathrm{eff}}^{2}$ with $\mu$ the $A-B$ reduced mass, $a_{\mathrm{eff}}=d$, and assuming that the $A$ atoms are degenerate fermions 
of Fermi momentum $k_{F}$ with $k_{F} d<1$; the constant $C_{\operatorname{dim}}$ is $6 \times 10^{-5}$ for the $A={ }^{6} \mathrm{Li}, B={ }^{87} \mathrm{Rb}$ case, resulting for $k_{F} d<1 / 2$ in a dimer formation rate much smaller than e.g. the $B$ fluorescence rate $\Gamma_{\text {fluo }}^{B}$ due to the lattice [30]. The calculation of the actual dimer formation rate in our model, taking into consideration the trapping of the $B$ atoms, requires the solution of a three-body problem with no center-of-mass separability, which is beyond the scope of the present work.

A second undesired effect is the one of gravity. If the lattice is arranged to be stationary in a free-falling frame, this frame having initially an upward velocity component $V$ in the lab frame and finally a downward velocity component $V$, the overall vertical motion of the lattice in such a fountain-like experiment is less than $3 \mathrm{~cm}$ for a total time of $150 \mathrm{~ms}$. Longer times may be obtained if one compensates gravity, e.g. by a using the inflexion point of the optical potential produced by a far-detuned Gaussian laser beam, or by using electro-optical potentials [59]. A drastic solution is of course to perform the experiment in a micro-gravity environment [60].

\section{LOCALIZED STATES}

The Ioffe-Regel criterion Eq. (1) is considered as a necessary condition to achieve strong localization [5]. It is simple to check that the setup that we consider can satisfy this criterion for experimentally reasonable parameters. As we will see in Sec. IV the effective scattering length presents confinement induced resonances that allow one to reach the unitary regime for the interaction of the matter wave with a trapped $B$ atom, with the maximal cross-section $\sigma=4 \pi / k^{2}$. In this case, the Ioffe-Regel criterion reads:

$$
k d \lesssim(4 \pi p)^{1 / 3}
$$

which for a filling factor $p=0.1$ yields $k \lesssim 1 / d=k_{L} / \pi$, achievable with sub-recoil laser cooling techniques, or with a low density degenerate Fermi gas with a Fermi wavevector $k_{F}<k_{L} / \pi$.

Since the Ioffe-Regel criterion is not proved to be sufficient, we numerically investigate in this section the possibility for the disordered model Hamiltonian Eq. (17) to lead to matter wave localization. In particular, we shall find that the unitary regime $a_{\mathrm{eff}}=\infty$ is not the most favorable one.

\section{A. How to find localized wavefunctions ?}

A criterion of strong localization presented by Kramer and MacKinnon [5] for electrons in a solid consists in showing that, at the Fermi energy $E=E_{F}$, off-diagonal elements of the resolvent $\mathcal{G}=\left(E+i 0^{+}-\mathcal{H}\right)^{-1}$ in real space decrease exponentially with the distance between the two considered points.
For our model Hamiltonian, the calculation of the matrix elements of the resolvent is straightforward to implement numerically, using a technique well known for scalar light waves in a gas of scatterers [23, 24]. These matrix elements are indeed given in presence of $N$ pointlike scatterers by

$$
\begin{aligned}
\left\langle\mathbf{r}|\mathcal{G}| \mathbf{r}^{\prime}\right\rangle & =g_{0}\left(\mathbf{r}-\mathbf{r}^{\prime}\right)+\frac{2 \pi \hbar^{2}}{m_{A}} \times \\
& \times \sum_{j, l} g_{0}\left(\mathbf{r}-\mathbf{r}_{j}\right)\left[M^{-1}\right]_{j l} g_{0}\left(\mathbf{r}_{l}-\mathbf{r}^{\prime}\right) .
\end{aligned}
$$

Here $g_{0}$ is the propagator in free space of a particle of positive energy $E \equiv \hbar^{2} k^{2} / 2 m_{A}, k>0$ :

$$
g_{0}\left(\mathbf{r}-\mathbf{r}^{\prime}\right) \equiv\left\langle\mathbf{r}\left|\mathcal{G}_{0}\right| \mathbf{r}^{\prime}\right\rangle=-\frac{m_{A}}{2 \pi \hbar^{2}} \frac{e^{i k\left|\mathbf{r}-\mathbf{r}^{\prime}\right|}}{\left|\mathbf{r}-\mathbf{r}^{\prime}\right|}
$$

and we have introduced the $N \times N$ matrix $M$ :

$$
M=\frac{I}{a_{\mathrm{eff}}}+M^{\infty}
$$

where $I$ is the identity matrix and $M^{\infty}$ a complex symmetric (not hermitian) matrix with elements defined by

$$
M_{j l}^{\infty}=\left\{\begin{array}{cl}
\exp \left(i k\left|\mathbf{r}_{j}-\mathbf{r}_{l}\right|\right) /\left|\mathbf{r}_{j}-\mathbf{r}_{l}\right| & \text { if } j \neq l, \\
i k & \text { if } j=l .
\end{array}\right.
$$

The exact calculation of the resolvent in coordinates space is in this way reduced to the inversion of the $N \times N$ matrix $M$ [31].

We have implemented the criterion by Kramer and MacKinnon for a variable energy $E$, and indeed we found an exponential decay of $\left|\left\langle\mathbf{r}|\mathcal{G}| \mathbf{r}^{\prime}\right\rangle\right|^{2}$ for sufficiently low energies. However, as shown in the appendix A this rapid decay is not a proof of localization but may be due to the fact that $E$ is in a spectral gap of the system [32].

The most direct way to prove localization is to exhibit stationary states that are 'localized' inside the disordered potential, that is with a wave function strongly peaked inside the scattering medium, decreasing exponentially towards the borders of the scattering medium. To this end, we use the fact that the wave function

$$
\phi\left(\mathbf{r} ; \mathbf{r}_{0}\right) \equiv \operatorname{Im}\left\langle\mathbf{r}\left|\mathcal{G}\left(E+i 0^{+}\right)\right| \mathbf{r}_{0}\right\rangle,
$$

when not identically zero, is an exact eigenstate of $\mathcal{H}$ with energy $E$, whatever the arbitrary location of its center $\mathbf{r}_{0}$ [33]. Here we take $E>0$ so that $\phi\left(\mathbf{r} ; \mathbf{r}_{0}\right)$ belongs to the continuum of the energy spectrum of $\mathcal{H}$, like the scattering states. A useful expression of $\phi$ is then

$$
\phi\left(\mathbf{r} ; \mathbf{r}_{0}\right)=A \operatorname{Im}\left[\frac{e^{i k\left|\mathbf{r}-\mathbf{r}_{0}\right|}}{\left|\mathbf{r}-\mathbf{r}_{0}\right|}+\sum_{j=1}^{N} d_{j} \frac{e^{i k\left|\mathbf{r}-\mathbf{r}_{j}\right|}}{\left|\mathbf{r}-\mathbf{r}_{j}\right|}\right]
$$

where $A=-m_{A} /\left(2 \pi \hbar^{2}\right)$ is a constant factor and $d_{j}=$ $-\sum_{l}\left(M^{-1}\right)_{j l} \exp \left(i k\left|\mathbf{r}_{l}-\mathbf{r}_{0}\right|\right) /\left|\mathbf{r}_{l}-\mathbf{r}_{0}\right|$. In practice, we choose $\mathbf{r}_{0}$ inside the scattering medium; to see if $\phi$ is localized or not, one just has to compare the values of $\phi$ inside and outside the scattering medium; one can even watch how the modulus of $\phi$ decays inside the medium. 


\section{B. Application of the proposed technique in 1D: analytical results}

We test the proposed technique in $1 \mathrm{D}$, where analytical results can be obtained. We assume that a quantum particle of mass $m$ interacts in $1 \mathrm{D}$ with $N$ pointlike scatterers that are randomly distributed in the interval $x \in[-L / 2, L / 2]$. Using the transfer matrix formalism, as detailed in the appendix B we can obtain exact expressions for the matrix elements of the resolvent $\psi(x) \equiv\left\langle x\left|\mathcal{G}\left(E+i 0^{+}\right)\right| 0\right\rangle$ as a function of $x$. Assuming that there is no scatterer in $x=0$, we obtain

$$
\psi(0)=\frac{m}{i \hbar^{2} k} \frac{\left(1+r_{-}\right)\left(1+r_{+}\right)}{1-r_{-} r_{+}}
$$

where $k>0$ is such that $E=\hbar^{2} k^{2} / 2 m, r_{-}$is the complex reflection coefficient for the set of scatterers in the halfspace $x<0$ oriented from $x=0$ to $x=-\infty$, and $r_{+}$is the complex reflection coefficient for the set of scatterers in the half-space $x>0$ oriented from $x=0$ to $x=+\infty$. In the half-space $x>L / 2$ we also have a simple expression:

$$
\psi(x)=\frac{m}{i \hbar^{2} k} \frac{t_{+}\left(1+r_{-}\right)}{1-r_{-} r_{+}} e^{i k x},
$$

where $t_{+}$is the transmission coefficient of the set of scatterers in the half-space $x>0$ oriented from 0 to $+\infty$. A similar expression holds for $x<-L / 2$, see appendix B

Using Furstenberg theorem [34], we know that in the thermodynamic limit $L \rightarrow+\infty$, with a fixed density of scatterers, the modulus of the transmission coefficients tends exponentially to zero,

$$
\left|t_{+}\right| \sim\left|t_{-}\right| \sim|t| \propto e^{-L / 2 \xi}
$$

where $\xi$ is the localization length, so that the modulus of $r_{+}$and $r_{-}$tends to one. We then find that the wavefunction $\phi(x)=\operatorname{Im} \psi(x)$ outside the scattering medium is of the order of $|t|$. To calculate $\phi$ inside the medium, we first take, to zeroth order in $|t|,\left|r_{+}\right|=\left|r_{-}\right|=1$ : Eq. (15) then leads to a purely real $\psi(0)$ (of order unity), that is to a vanishing $\phi(0)$. Going to next order, $\left|r_{ \pm}\right| \simeq 1-\left|t_{ \pm}\right|^{2} / 2$ leads to $|\phi(0)| \sim|t|^{2}$. For a generic value of $k$, the wavefunction $\phi(x)$ has therefore the behavior depicted in Fig 2].

However, for specific values $k_{0}$ of $k$, the above reasoning is incorrect. Assume that $k$ is such that $r_{+} r_{-}$is a real and positive number. Then the denominator $1-r_{+} r_{-}$of Eqs. 15 16 is extremely small, of order $|t|^{2}$. This leads to $|\phi(x)|$ decreasing exponentially from $x=0$ outwards, from a value $\sim 1 /|t|^{2}$ to a value $\sim 1 /|t|$ [35], as depicted in Fig 2 $\mathrm{p}$. In this case, $\phi(x)$ corresponds to a 'localized' state inside the medium.

Strictly speaking, since we consider disorder over a finite region, $\phi(x)$ is not localized: the exponential decrease of the envelope stops outside the scattering medium, so that $\phi(x)$ is not square integrable. The state $\phi(x)$ rather corresponds to a resonance, whose lifetime is of the order of $\hbar$ over the energy width of the resonance. In practice, $\phi$ can be considered as localized if the resonance lifetime is much longer than the duration of the experiment. Assuming that the phase of $r_{+} r_{-}$varies linearly with $k$ close to $k_{0}$, one finds that this 'localized' state is present on a narrow interval in $k$ of width $\propto|t|^{2}$, so that its energy width, or equivalently its inverse lifetime, scales as

$$
\Gamma \propto \frac{\left|t_{+}\right|^{2}+\left|t_{-}\right|^{2}}{2} \sim|t|^{2} \propto e^{-L / \xi} .
$$

This illustrates how the proposed technique, defined in Eq. (13), gives access to localized wavefunctions.
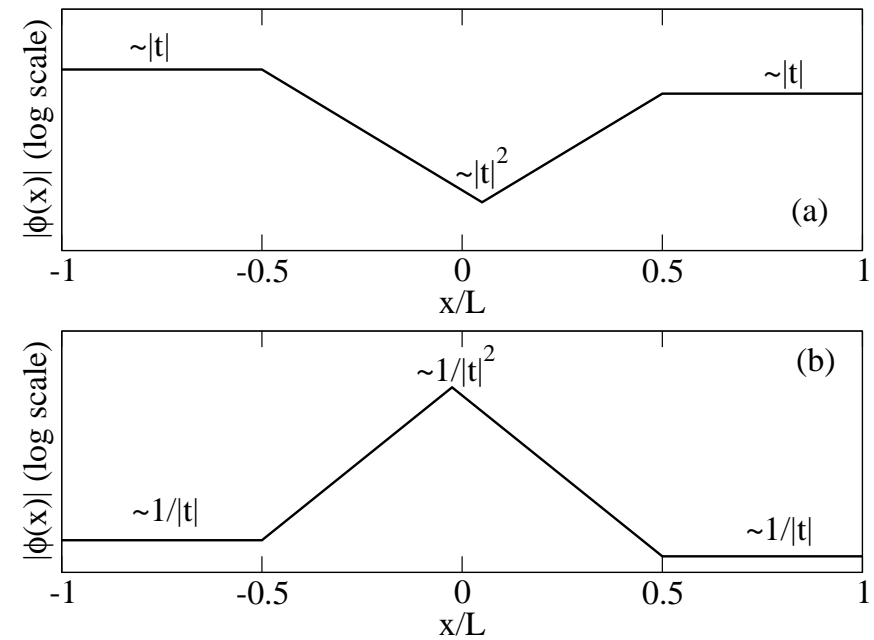

FIG. 2: Schematic view of the behavior of the wavefunction $\phi(x)=\operatorname{Im}\langle x|\mathcal{G}| 0\rangle$ in presence of a $1 \mathrm{D}$ scattering medium of length $L$ much larger than the localization length $\xi$, so that the modulus of the transmission coefficient for each half of the medium $\left|t_{+}\right|,\left|t_{-}\right| \sim|t| \propto e^{-L / 2 \xi} \ll 1$. (a) For a generic positive energy: the wavefunction decreases exponentially inside the medium, being of modulus $\sim|t|$ out of the medium and $\sim|t|^{2}$ in the center $x=0$ of the medium. (b) For specific values of the energy, the wavefunction is 'localized' inside the medium: its modulus decreases from $\sim 1 /|t|^{2}$ in $x=0$ to $\sim 1 /|t|$ for $|x|>L / 2$.

\section{Application of the proposed technique in 3D: numerical results}

We now present numerical results obtained for a single realization of a random potential obtained by a Monte Carlo generation of the positions of a finite number $N$ of scatterers at the nodes of a cubic lattice with a given filling factor $p$.

In Fig. 3 we plot the ratio of the square of the amplitudes of $\phi$ inside and outside the scattering medium, for $N \simeq 900$ scatterers and a filling factor $p=0.1$. In order to avoid a choice that might pick a node of $\phi(\mathbf{r})$, we 


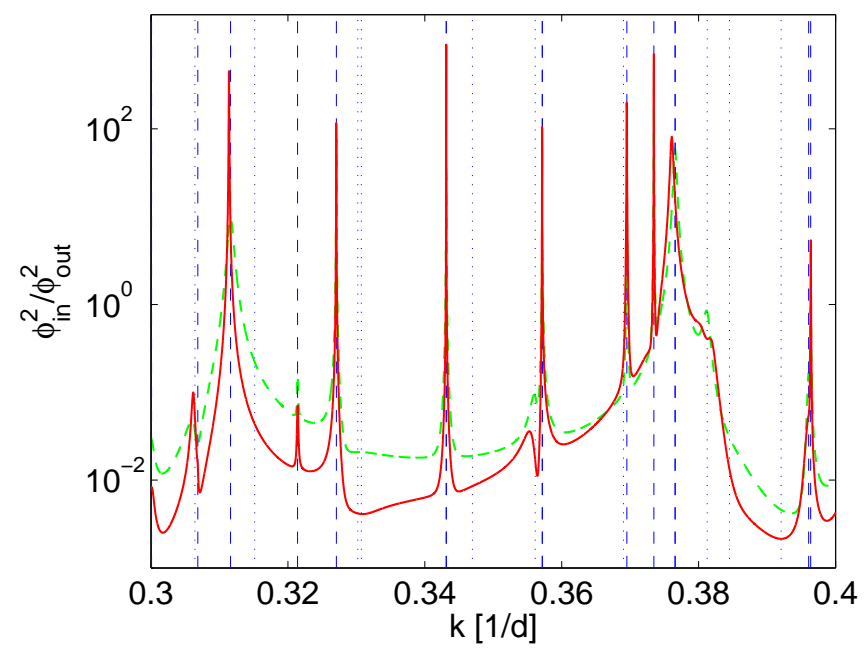

FIG. 3: (Color online) Ratio of the values of $\phi^{2}$ in the center and outside the scattering medium (see text) as a function of $k=\left(2 m_{A} E\right)^{1 / 2} / \hbar$, with $a_{\text {eff }}=d$, for a given realization of the disorder with $N \approx 900$ scatterers that occupy the nodes of a cubic lattice with 21 sites per side, with a filling factor $p=0.1$. The value of $\phi_{\text {out }}^{2}$ is calculated as explained in [36] (red solid curve) or with the extrapolation from the far field behavior, Eq. (20) with $r=R=20 d$ (green dashed curve). The energy intervals where the matter wave significantly penetrates the scattering medium correspond to the narrow peaks in this figure. We have checked that the wave function is actually spatially localized in such an energy interval. The vertical lines mark the locations of the resonances obtained by the spectral method: dashed lines for the very long lived resonances $\left(\Gamma<6 \times 10^{-5} E_{r}^{A} / \hbar\right)$ and dotted lines for the broader resonances. The temporal decay rate $\Gamma$ is obtained from Eq. 23).

plot $\phi_{\text {in }}^{2} / \phi_{\text {out }}^{2}$, where both numerator and denominator are averaged over a few points 36]. The graph reveals that the phenomenology is similar to the $1 \mathrm{D}$ case: one has generically $\phi_{\text {in }}^{2} \ll \phi_{\text {out }}^{2}$, except for narrow energy intervals, corresponding to the peaks in the figure, where the matter wave can significantly penetrate the scattering medium. We have verified for a large number of peaks that the wavefunction $\phi$ is indeed 'localized' inside the medium.

We illustrate this phenomenology for a generic value of $k$, and for one that corresponds to a peak in the function $\phi_{\text {in }}^{2} / \phi_{\text {out }}^{2}$ taking now a larger number of scatterers $N \simeq 3400$. As can be seen in Fig. 4 in the generic case $\phi(\mathbf{r})$ decays essentially exponentially when entering the medium, whereas for the specific value of $k$ the wavefunction decays essentially exponentially from the medium center towards the outside. What is the associated localization length $\xi$ ? If one takes $\phi \propto \exp \left(-\left|\mathbf{r}-\mathbf{r}_{0}\right| / \xi\right)$, one obtains from a fit the estimate $\xi \sim d[37$.
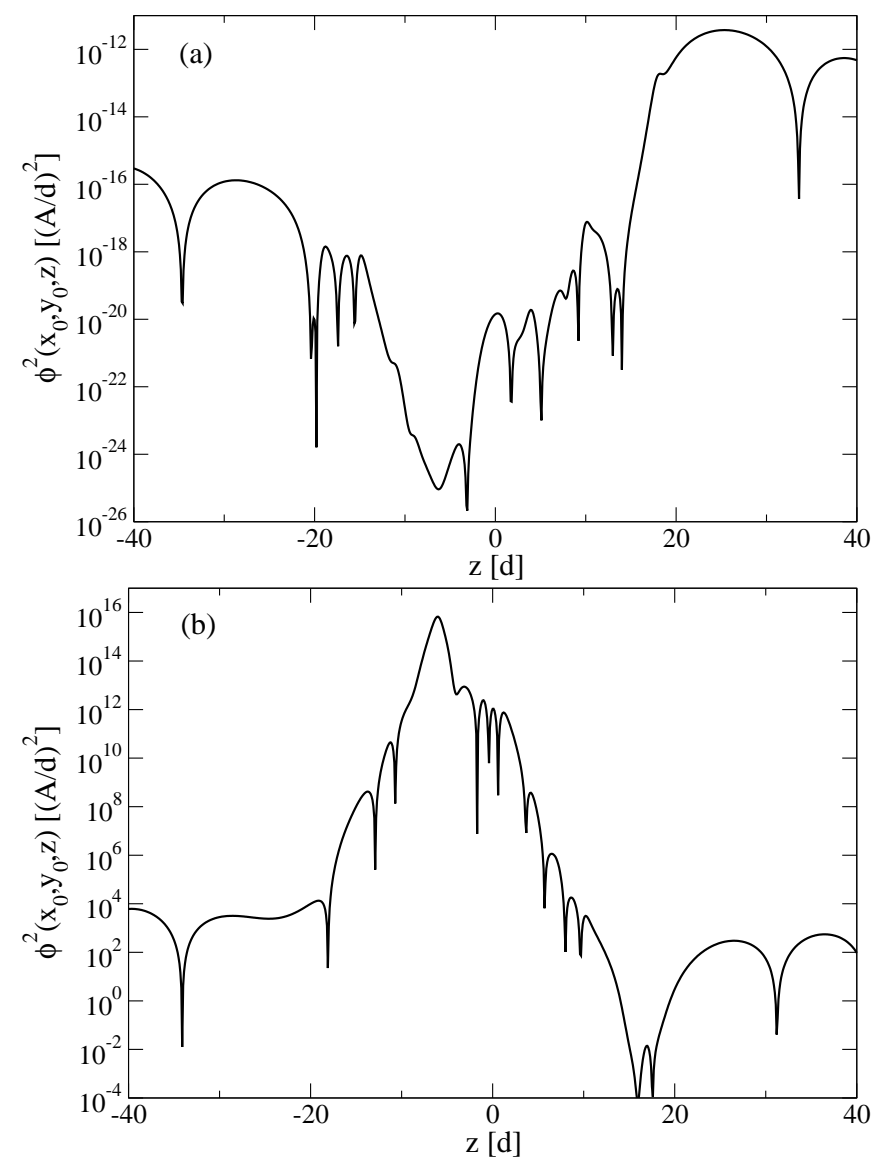

FIG. 4: Plot of $\phi(\mathbf{r})^{2}$ along the straight line passing through $\mathbf{r}_{0}$ and parallel to $z$ axis, for two values of $k$, (a) a generic value $k=0.3 / d$ and (b) a value $k=0.350134274724 / d$ corresponding to a peak for $\phi_{\text {in }}^{2} / \phi_{\text {out }}^{2}$ as a function of $k$ (in the spirit of Fig 31). Note the similarity with the 1D case sketched in Fig 2 In (a) the position $\mathbf{r}_{0}$ is close to the center of the scattering medium: $\mathbf{r}_{0}=(d / 2,-d / 2,-d / 2)$; in (b) it is close to the 'center' of the localized state: $\mathbf{r}_{0}=(15 d / 2,-3 d / 2,-11 d / 2)$. The effective scattering length is given by $d / a_{\text {eff }}=1.20530122302$. To get a clear evidence of the exponential decay of $\phi$, we used a larger scattering medium than in Fig. 3.3400 atoms on the lattice within a sphere of radius $20 d$, with an occupation probability $p=0.1$.

\section{Comparison with a spectral technique}

In this subsection, we adopt a different point of view on localization [38], based on the properties of the analytic continuation of the resolvent $\mathcal{G}(z)=1 /(z-\mathcal{H})$ from the half-plane $\operatorname{Im} z>0$ to the half-plane $\operatorname{Im} z<0$, across the branch cut $\operatorname{Im}(z)=0, \operatorname{Re}(z)>0$ corresponding to the continuous spectrum of the Hamiltonian. This analytic continuation can have poles in $z=E_{0}-i \hbar \Gamma / 2$, where $E_{0}$ and $\Gamma$ are positive (see Fig. 可): these poles correspond to resonances. The lifetime of the state associated to the resonance is given by $\Gamma^{-1}$.

The key property of localized states is that they cor- 


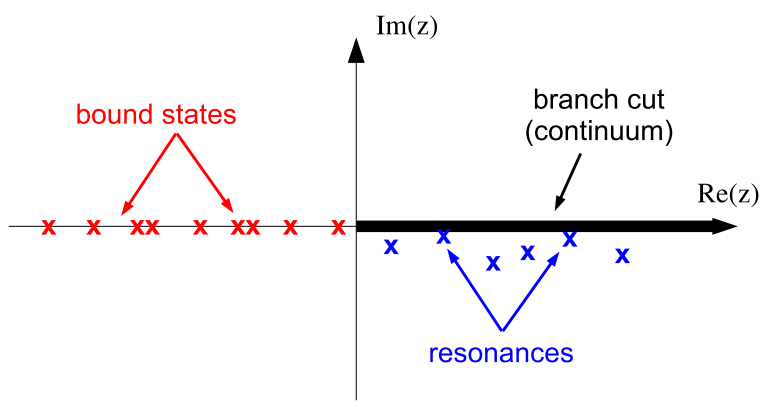

FIG. 5: (Color online) Analytic properties of the resolvent $\mathcal{G}(z)=1 /(z-\mathcal{H})$ in the complex plane $z$. The resolvent has poles on the negative side of the real axis, corresponding to bound states, and a branch cut on the positive side of the real axis, corresponding to the continuum. The analytic continuation of the resolvent across the branch cut from $\operatorname{Im} z>0$ to $\operatorname{Im} z<0$ may present a discrete set of poles in the fourth quadrant: the associated states are the resonances of the system.

respond to resonances with a decay rate $\Gamma$ that tends to zero in the limit of an infinite extension of the disorder, so that they become in this limit square integrable stationary states of the Hamiltonian. We expect that the poles associated to these narrow resonances will leave signatures on the real axis, in the form of eigenvalues of the matrix $M$ with vanishing real part and tiny imaginary part, for an energy $E=\hbar^{2} k^{2} / 2 m_{A}$ close to the real part $E_{0}$ of the poles. As opposed to the poles of $\mathcal{G}$, the eigenvalues of $M$ can be calculated in a straightforward manner, and in Fig. 3 we show with vertical lines the values of $k$ for which the matrix $M$ has a purely imaginary eigenvalue: the ones with the smallest imaginary parts (dashed vertical lines) are in good correspondence with the narrow peaks in $\phi_{\text {in }}^{2} / \phi_{\text {out }}^{2}$.

Now, for a value of $k$ within the width of a peak in Fig 3 we give an analytical argument relating the spatial decay of $\phi(\mathbf{r})$ to the presence of a tiny eigenvalue $m_{0}$ of $M$ of modulus much smaller than the other eigenvalues. In the large $r$ limit, Eq. (14) reduces to the far field expression

$$
\phi\left(\mathbf{r} ; \mathbf{r}_{0}\right)=A \operatorname{Im}\left[\frac{e^{i k r}}{r}\left(e^{-i k \mathbf{n} \cdot \mathbf{r}_{0}}+\sum_{j=1}^{N} d_{j} e^{-i k \mathbf{n} \cdot \mathbf{r}_{j}}\right)\right],
$$

with $\mathbf{n}=\mathbf{r} / r$. As shown in the appendix $\mathbb{C}$ the sum over $j$ in the right hand side is typically $\left(\operatorname{Im} 1 / m_{0}^{*}\right)^{1 / 2}$ times larger than the first term so that the angular average of $\phi^{2}$ is given, apart from oscillating terms $\sim e^{2 i k r} / r^{2}$, by

$$
\left\langle\phi\left(\mathbf{r} ; \mathbf{r}_{0}\right)^{2}\right\rangle_{\mathbf{n}} \sim A^{2} \frac{\vec{d}^{*} \cdot(\operatorname{Im} M) \vec{d}}{2 k r^{2}} .
$$

This allows to estimate $\phi_{\text {out }}$ by extrapolating this far field expression down to $r=R$, where $R$ is a distance of order the size of the scattering medium [39], see the green dashed curve in Fig 3 We now need to estimate the wavefunction inside the medium. Close to the scattering center located in $\mathbf{r}_{j}$, one finds $\phi \sim A \operatorname{Im} d_{j} /\left|\mathbf{r}-\mathbf{r}_{j}\right|$. This suggests that the $d_{j}$ are also localized, in the sense that they decrease rapidly from a center in the medium outwards: this we have checked numerically. Then, averaging spatially over a small volume $l^{3}, l$ being smaller than the mean distance between scatterers, one finds that $\phi_{\mathrm{in}}^{2}$ is at most of the order of $(A / l)^{2} \max _{j}\left|\operatorname{Im} d_{j}\right|^{2}$, a value reached when $\mathbf{r}_{0}$ is close to the 'center' of the localized wavefunction. We reach the estimate

$$
\frac{\phi_{\text {in }}^{2}}{\phi_{\text {out }}^{2}} \sim \frac{R^{2}}{l^{2}} \frac{\max _{j}\left|\operatorname{Im} d_{j}\right|^{2}}{\vec{d}^{*} \cdot(\operatorname{Im} M / k) \vec{d}} .
$$

In the right hand side, the first factor has a geometrical origin whereas the second one is sensitive to matter wave interference effects due to multiple scattering on the $B$ atoms: since the matrix elements of $\operatorname{Im} M / k$ are of the order of unity, only interference effects can indeed lead to a very small expectation value of this matrix. Using arguments detailed in the appendix $[$ we ultimately arrive at

$$
\frac{\phi_{\mathrm{in}}^{2}}{\phi_{\mathrm{out}}^{2}} \sim \frac{R^{2}}{l^{2}} \operatorname{Im}\left[\frac{k}{m_{0}^{*}}\right],
$$

which links the spatial decay of the wavefunction to the smallness of an eigenvalue of the matrix $M$. A useful application of this formula is to give the shape of the resonances in Fig 3 We linearize the $k$ dependence of $m_{0}$ around the value $k_{0}$ such that $m_{0}$ is purely imaginary: $m_{0}(k) \simeq \beta\left(k-k_{0}\right)+i \alpha$, where $\alpha>0$. Anticipating Fig. [6 it appears that the imaginary part of an eigenvalue of $M^{\infty}$, when tiny for $k=k_{0}$, remains tiny for even lower values of $k$, so that the derivative of $\operatorname{Im} m_{0}$ is also tiny; since the real part varies on the contrary over an interval of width $1 / d$, its derivative is not extremely small: $\beta$ is essentially real [40]. Eq. (22) then leads to a Lorentzian shape of the peaks in Fig 3 in agreement with the numerics. Since the peaks have a width much narrower than $k_{0}$, this also leads to a Lorentzian dependence with the energy $E=\hbar^{2} k^{2} / 2 m_{A}$, with a half-width at half maximum [41]:

$$
\frac{\hbar \Gamma}{2} \simeq \frac{\hbar^{2} k_{0}}{m_{A}} \frac{\operatorname{Im} m_{0}\left(k_{0}\right)}{\operatorname{Re} m_{0}^{\prime}\left(k_{0}\right)} \propto \operatorname{Im} m_{0} .
$$

When combined to Eq. (22), this leads to a formula nicely relating the inverse lifetime $\Gamma$ of the localized state to its spatial decay:

$$
\Gamma \propto \frac{\phi_{\text {out }}^{2}}{\phi_{\text {in }}^{2}}\left(k=k_{0}\right) .
$$

If one then assumes a state with a wavefunction localized close to the center of the medium and decaying exponentially as $e^{-r / \xi}$ away from the center, Eq. (22) leads to $\operatorname{Im} m_{0} \propto e^{-L / \xi}$, where $L$ is the diameter of the scattering medium, and Eq. (24) leads to a resonance energy 
width $\Gamma \propto e^{-L / \xi}$, thus obeying the same scaling as in 1D, see Eq. (18).

An important practical consequence of the present spectral approach is to give at once the range of values of $a_{\text {eff }}$ for which one can hope to have localized states. For a given value of $k, M$ will have a tiny purely imaginary eigenvalue if $1 / a_{\text {eff }}$ is opposite to the real part of an eigenvalue of $M^{\infty}$ with a very small imaginary part, see Eq. (11). In Fig. [6] the eigenvalues of $M^{\infty}$ are shown as points in the complex plane, for various values of the incoming wave number $k$ and for two realizations of the disorder with widely different densities of scatterers. We observe that, as the incoming wave number $k$ decreases below the inverse of the mean distance between the scatterers $p^{1 / 3} / d$, many eigenvalues acquire an extremely small imaginary part and accumulate in the region $\operatorname{Re} m^{\infty} \sim-2 p^{1 / 3} / d$ (see the green dashed lines in Fig. [6), similarly to earlier calculations for light waves 42.

In a matter wave experiment, this suggests to tune $a_{\text {eff }}^{-1}$ to a value close to $2 p^{1 / 3} / d$ (as we have done in Fig [3): this might be achieved in practice by using a Feshbach resonance, as shown in the next section. In this way, as $k$ decreases below $2 p^{1 / 3} / d$, one obtains a very large sequence of values of $k$ such that $M$ has a tiny purely imaginary eigenvalue, that is one may have access to a large number of localized states. This is illustrated in Fig [

Finally, the representation in Fig [ is also useful to understand what happens in the low energy limit, $k \rightarrow 0$ It shows that, for each eigenvalue $m^{\infty}$ of the matrix $M$, $\operatorname{Re} m^{\infty}$ and $\operatorname{Im} m^{\infty} / k$ have a finite limit [43]; a numerical inspection reveals that some of the eigenstates of $M^{\infty}(k=0)$, having a tiny value of $\lim _{k \rightarrow 0} \operatorname{Im} m^{\infty} / k$, are localized. Can these localized states in the zero energy limit be accessed in a real experiment? For a given realization of disorder, this would require that $a_{\mathrm{eff}}$ be tuned exactly to one of the corresponding values of $-1 / \operatorname{Re} m^{\infty}$, which is unrealistic.

In the same way, it would be very difficult to adjust $a_{\text {eff }}$ to hit, at a given value of $k$, one of the peaks in Fig 0 Fortunately, in a real experiment, $a_{\text {eff }}$ is fixed and a broad interval of $k$ can be populated by the atomic wavepackets; then, Fig [6] shows that the real parts of the eigenvalues of $M^{\infty}$ with tiny imaginary parts are increasing functions of $k$, so that they have a chance to cross the value $-1 / a_{\text {eff }}$ for some value of $k$ within the experimentally populated interval, and to lead to a peak in Fig 3 and to an observable localized state in the experiment.

To conclude this section, it is useful to discuss the number of localized states that can be supported by a finite size scattering medium, to have an idea of the number of atoms that may populate these localized states in a real experiment, and to have an estimate of the number of states that can be considered as localized for a given duration of the experiment. To this end, we present in Fig 8 an histogram giving the number of purely imaginary eigenvalues of $M$ per class of inverse lifetimes $\Gamma$. It
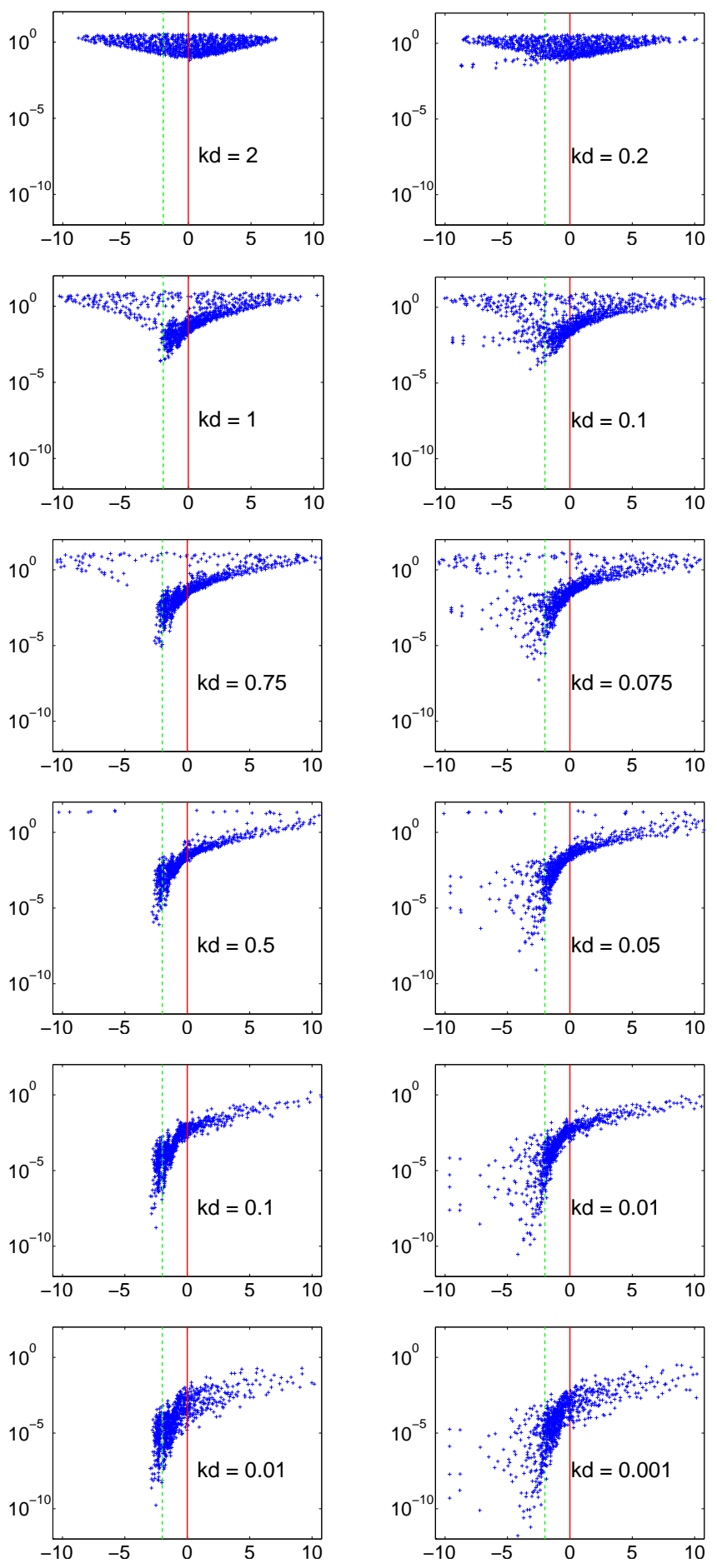

FIG. 6: (Color online) Representation in the complex plane of the eigenvalues $m^{\infty}$ of $M^{\infty}$ for different values of the wave number $k$ of the matter wave. The eigenvalues of $M$ are then simply deduced from these eigenvalues by a shift of $1 / a_{\text {eff }}$ along the real axis. Left column: cubic lattice with 21 sites/side, $p=0.1$ and 872 scatterers; the inverse mean distance between scatterers is $p^{1 / 3} / d \simeq 0.46 / d$ (same realization of disorder as in Fig. (3). Right column: cubic lattice with 209 sites/side, $p=10^{-4}$ and 878 scatterers; the inverse mean distance between scatterers is $p^{1 / 3} / d \simeq 0.046 / d$. The green dashed lines mark the values $\operatorname{Re}\left(m^{\infty}\right)=-2 p^{1 / 3} / d$. The real axis is in units of $p^{1 / 3} / d$, and common values of $k d / p^{1 / 3}$ are taken in both columns, so as to reveal a possible universality in the low $p$ limit. The imaginary axis is in units of $k$, as justified by Eq. 222. 


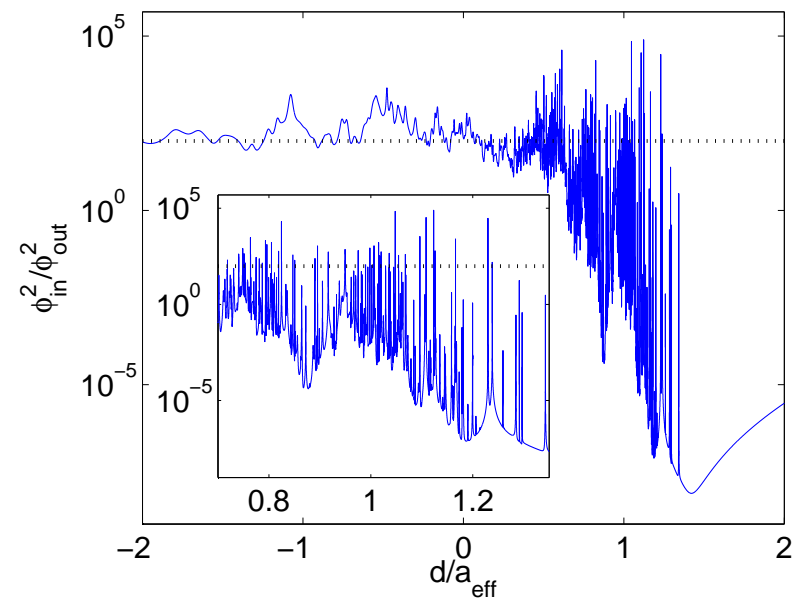

FIG. 7: (Color online) For a fixed positive energy, corresponding to $k=0.35 / d$, ratio of the values of $\phi^{2}$ in the center and outside the scattering medium as a function of the effective scattering length $a_{\text {eff }}$ [36]. The dashed line gives the result for $a_{\text {eff }}=0$. The same realization of disorder is used as in Fig [3 The inset is a magnification of the region $a_{\mathrm{eff}} \sim d$.

is apparent on this figure how an increase of the volume of the scattering medium (here by a factor $\sim 3.3$ ) leads to both an increase in the total number of localized states (for a given lifetime) and to the appearance of a tail of states of significantly longer lifetimes (here by about two orders of magnitude).

\section{EFFECTIVE INTERACTION}

We will solve here the two-body problem of a free atom $A$ scattering on a single trapped particle $B$ prepared in the ground state of a harmonic oscillator potential. In the limit of vanishing incoming kinetic energy of the $A$ particle, this gives access to the effective scattering length introduced in Eq. (5), which is the key parameter of our model Hamiltonian with disorder.

\section{A. Calculation of the $A-B$ scattering amplitude}

The Hamiltonian for a free $A$ particle and a harmonically trapped $B$ one is, in the absence of $A-B$ interaction,

$$
H_{0}=-\frac{\hbar^{2} \Delta_{A}}{2 m_{A}}-\frac{\hbar^{2} \Delta_{B}}{2 m_{B}}+\frac{1}{2} m_{B} \omega^{2} r_{B}^{2}
$$

It admits the $s$-wave solution 44

$$
\psi_{0}\left(\mathbf{r}_{A}, \mathbf{r}_{B}\right)=\frac{\sin \left(k r_{A}\right)}{k r_{A}} \phi_{0}\left(r_{B}\right)
$$

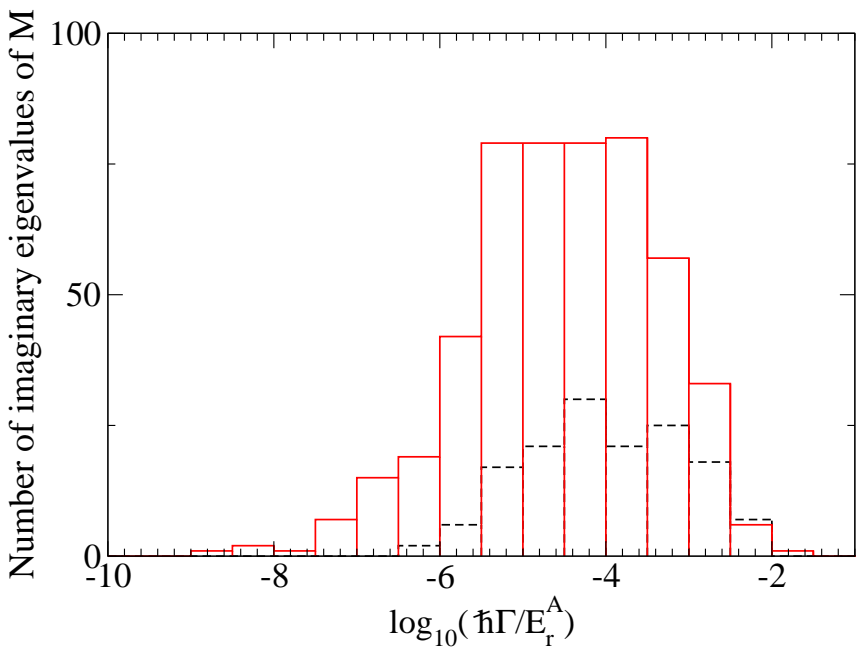

FIG. 8: (Color online) For a single realization of disorder, histogram giving the number of imaginary eigenvalues of $M$ per class of inverse lifetimes $\Gamma^{-1}$. The filling factor of the lattice is $p=0.1$ and the effective scattering length is $a_{\mathrm{eff}}=$ d. Black dashed histogram: $N=872$ scatterers within a cube with 21 sites per side. Red solid line histogram: $N=$ 2985 scatterers within a cube with 31 sites per side. These histograms were constructed by a dichotomy search of the values $k_{0}$ of the momentum $k \in[0,1 / d]$ such that the matrix $M$ has a purely imaginary eigenvalue; the associated lifetime was then calculated with Eq. (23). Eigenvalues with negative values of $\Gamma$ are of course not included (see [41]). The decay rate $\Gamma$ is given in units of the recoil angular frequency $E_{r}^{A} / \hbar=$ $\hbar k_{L}^{2} / 2 m_{A}$ of the species $A$. In practice, only the eigenvalues with small enough values of $\Gamma$ are expected to produce an observable resonance in $\phi_{\text {in }}^{2} / \phi_{\text {out }}^{2}$ as a function of $k$, see Fig 3 If the matter wave is made of atoms of ${ }^{6} \mathrm{Li}$, a duration of the experiment of $0.3 \mathrm{~s}$ corresponds to a minimal observable inverse lifetime of $\Gamma \sim 10^{-5} E_{r}^{A} / \hbar$ for an optical lattice laser wavelength of $779 \mathrm{~nm}$.

which represents a spherical matter wave $(A)$ of wave vector $k$ and a particle $(B)$ in the ground state of the harmonic potential, $\phi_{0}\left(r_{B}\right)=\exp \left(-r_{B}^{2} / 2 a_{\mathrm{ho}}^{2}\right) /\left(\sqrt{\pi} a_{\mathrm{ho}}\right)^{3 / 2}$, with the harmonic oscillator length $a_{\mathrm{ho}}=\left(\hbar / m_{B} \omega\right)^{1 / 2}$. The state $\psi_{0}$ has an energy

$$
E=\frac{\hbar^{2} k^{2}}{2 m_{A}}+\frac{3}{2} \hbar \omega
$$

We model the $A$ - $B$ interaction with a regularized contact potential, which leads to the full Hamiltonian:

$$
H=H_{0}+g \delta\left(\mathbf{r}_{A}-\mathbf{r}_{B}\right) \frac{\partial}{\partial\left|\mathbf{r}_{A}-\mathbf{r}_{B}\right|}\left(\left|\mathbf{r}_{A}-\mathbf{r}_{B}\right| \ldots\right) .
$$

Here, the derivative is taken for a fixed value of the center of mass position $\mathbf{R}$ of the two particles, the coupling constant $g$ is expressed in terms of the reduced mass $\mu=m_{A} m_{B} /\left(m_{A}+m_{B}\right)$ and the free-space scattering length $a$ (relative to the $A-B$ interaction in the absence 
of the trapping potential) by

$$
g=\frac{2 \pi \hbar^{2} a}{\mu} .
$$

The Schrödinger equation $H \psi=E \psi$ can be reformulated equivalently in the integral form

$\psi\left(\mathbf{r}_{A}, \mathbf{r}_{B}\right)=\psi_{0}\left(\mathbf{r}_{A}, \mathbf{r}_{B}\right)+g \int \mathrm{d} \boldsymbol{\rho} G_{E}\left(\mathbf{r}_{A}, \mathbf{r}_{B} ; \boldsymbol{\rho}, \boldsymbol{\rho}\right) \psi_{\mathrm{reg}}(\rho)$

in terms of the two-particle retarded Green's function for the non-interacting Hamiltonian

$$
G_{E}=\frac{1}{E+i 0^{+}-H_{0}}
$$

and the regularized part of the two-particle wave function,

$$
\psi_{\mathrm{reg}}(\mathbf{R})=\left.\frac{\partial}{\partial\left|\mathbf{r}_{A}-\mathbf{r}_{B}\right|}\left[\left|\mathbf{r}_{A}-\mathbf{r}_{B}\right| \psi\left(\mathbf{r}_{A}, \mathbf{r}_{B}\right)\right]\right|_{\mathbf{r}_{A}=\mathbf{r}_{B}}
$$

where $\mathbf{R}=\left(m_{A} \mathbf{r}_{A}+m_{B} \mathbf{r}_{B}\right) /\left(m_{A}+m_{B}\right)$ and $\psi_{\text {reg }}(\mathbf{R})=$ $\psi_{\text {reg }}(R)$ since we consider $s$-wave scattering only. Insert-

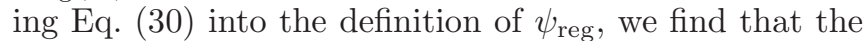
regularized part of the wave function satisfies an equation of the form

$$
\tilde{\psi}_{\mathrm{reg}}=\frac{I}{I-g \hat{O}} \tilde{\psi}_{0}
$$

where $\tilde{\psi}(R)=R \psi(R), \tilde{\psi}_{0}(R)=R \phi_{0}(R) \sin (k R) /(k R)$, and $\hat{O}$ is an integral operator independent of the scattering length $a$. The detailed derivation of this equation and the explicit form of $\hat{O}$ are presented in Appendix E

As we now show, the knowledge of $\tilde{\psi}_{\text {reg }}$ directly leads to the value of the scattering amplitude $f_{k}$ for the $A$ wave. Expanding $G_{E}$ on the basis of eigenstates $\left|\mathbf{k}_{A}, \mathbf{n}\right\rangle$ of $H_{0}$, and projecting in position space, one is able to calculate the integral over the wavevector $\mathbf{k}_{A}$ so that one is left with a sum over the vibrational states of the $B$ particle:

$$
\begin{aligned}
G_{E}\left(\mathbf{r}_{A}, \mathbf{r}_{B} ; \boldsymbol{\rho}, \boldsymbol{\rho}\right) & =\frac{2 m_{A}}{\hbar^{2}}\left[-\frac{e^{i k\left|\mathbf{r}_{A}-\boldsymbol{\rho}\right|}}{4 \pi\left|\mathbf{r}_{A}-\boldsymbol{\rho}\right|} \phi_{0}\left(\mathbf{r}_{B}\right) \phi_{0}(\boldsymbol{\rho})\right. \\
- & \left.\sum_{\mathbf{n} \neq 0} \frac{e^{-\kappa_{\mathbf{n}}\left|\mathbf{r}_{A}-\boldsymbol{\rho}\right|}}{4 \pi\left|\mathbf{r}_{A}-\boldsymbol{\rho}\right|} \phi_{\mathbf{n}}\left(\mathbf{r}_{B}\right) \phi_{\mathbf{n}}(\boldsymbol{\rho})\right] .
\end{aligned}
$$

The low-energy assumption (3) ensures that the only open exit channel corresponds to a $B$ particle in the ground vibrational state, and therefore that

$$
-\frac{\hbar^{2} \kappa_{\mathbf{n}}^{2}}{2 m_{A}}=\frac{\hbar^{2} k^{2}}{2 m_{A}}-\hbar \omega\left(n_{x}+n_{y}+n_{z}\right)<0, \forall \mathbf{n} \neq 0 .
$$

As a consequence, the terms of Eq. (34) involving excited states of the harmonic oscillator give exponentially vanishing contributions and can be neglected when $A$ is at a distance $\gg a_{\text {ho }}$ from the center of the harmonic well.
Expanding $\left|\mathbf{r}_{A}-\boldsymbol{\rho}\right| \simeq r_{A}-\boldsymbol{\rho} \cdot \mathbf{r}_{A} / r_{A}$ and substituting into Eq. (30), we get

$$
\psi\left(\mathbf{r}_{A}, \mathbf{r}_{B}\right) \stackrel{r_{A} \rightarrow \infty}{\simeq}\left[\frac{\sin \left(k r_{A}\right)}{k r_{A}}+f_{k} \frac{\exp \left(i k r_{A}\right)}{r_{A}}\right] \phi_{0}\left(r_{B}\right),
$$

where we have introduced the scattering amplitude

$$
f_{k}=-a \frac{m_{A}}{\mu} \int \mathrm{d} \rho \frac{\sin (k \rho)}{k \rho} \phi_{0}(\rho) \psi_{\mathrm{reg}}(\rho) .
$$

In the limit of zero-energy, the scattering amplitude defines the effective scattering length $a_{\mathrm{eff}}$ through

$$
a_{\text {eff }} \equiv-\lim _{k \rightarrow 0} f_{k}=a \frac{m_{A}}{\mu} \int \mathrm{d} \boldsymbol{\rho} \phi_{0}(\rho) \psi_{\text {reg }}^{k=0}(\rho) .
$$

We have solved numerically Eq. (33), and in Figs. 9. 111 we plot $a_{\text {eff }}$ as a function of $1 / a$ for different values of $m_{B} / m_{A}$. We choose $m_{B} / m_{A}=0.15,1,6.67$, corresponding to the physical cases of a mixture of $A={ }^{40} \mathrm{~K}$ and $B={ }^{6} \mathrm{Li}$, a mixture of two different internal states of atoms of the same species, and a mixture of $A={ }^{6} \mathrm{Li}$ and $B={ }^{40} \mathrm{~K}$, respectively. It is apparent that $a_{\text {eff }}$ presents a series of intriguing resonances, which can be used to tune $a_{\text {eff }}$ to a high value and whose physical original are discussed in $\dddot{\mathrm{IVB}}$

In Figs. 10] and 12 we also plot, in the case $m_{B}=m_{A}$, the behavior of the effective range $r_{e}$. It is defined as a coefficient in the low $k$ expansion of the inverse scattering amplitude:

$$
f_{k}^{-1}=-\left[a_{\mathrm{eff}}^{-1}+i k-r_{e} k^{2} / 2+\ldots\right] .
$$

The replacement of a trapped $B$ particle by a fixed pointlike scatterer, as done in Eq. (5), is allowed when the $k^{2}$ term in the above expansion is negligible, i.e. when

$$
\left|r_{e}\right| k^{2} \ll\left|a_{\mathrm{eff}}^{-1}+i k\right| .
$$

In the ideal regime for matter wave localization, $k<$ $a_{\mathrm{eff}}^{-1} \sim 1 / d$. Since $r_{e}$ is generally of order $a_{\mathrm{ho}} \ll d$, this condition Eq. (40) is satisfied. Note that $r_{e}$ diverges when $a_{\text {eff }} \rightarrow 0$, a generic phenomenon: expanding $f_{k}$ rather than $f_{k}^{-1}$ in powers of $k$,

$$
f_{k}=-a_{\mathrm{eff}}+i k a_{\mathrm{eff}}^{2}-\frac{1}{2} r_{e} a_{\mathrm{eff}}^{2} k^{2}+\ldots
$$

one generically expects that $r_{e} a_{\text {eff }}^{2}$ has a finite limit when $a_{\text {eff }} \rightarrow 0$. This was demonstrated analytically both for a square well [45] and a van der Waals interaction potential [46]. In this limit, Eq. (40) is thus violated for any finite $k$; however, this is not an ideal regime to obtain matter wave localization.

\section{B. Resonances of the effective scattering length}

We now show that the effective coupling constant experiences an infinite set of resonances due to the presence 


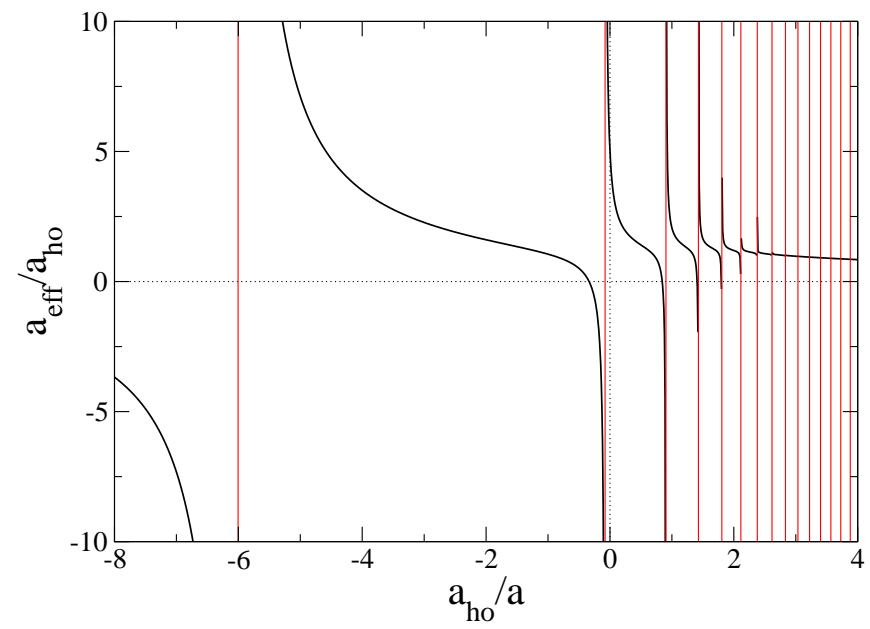

FIG. 9: (Color online) Effective scattering length $a_{\text {eff }}$ (continuous line) as a function of $a^{-1}$ for $m_{B} / m_{A}=0.15$ (trapped ${ }^{6} \mathrm{Li}$ and free ${ }^{40} \mathrm{~K}$ ). The vertical lines (red) mark the positions of the resonances (a step of $a_{\mathrm{ho}} / a=0.01$ is used to sample the curves, and some of the resonances are too narrow to be seen on the graph).

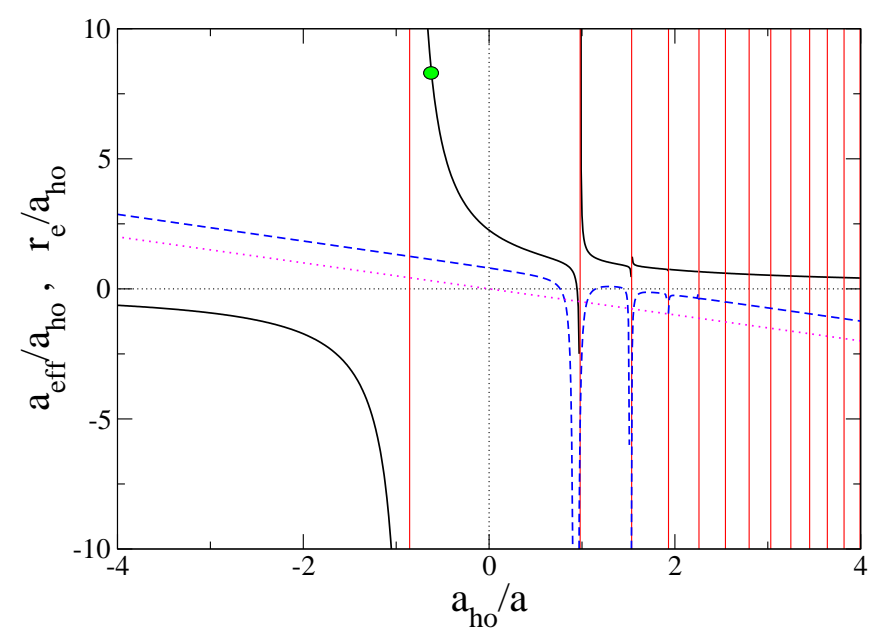

FIG. 10: (Color online) Same as Fig. 9 for two particles of equal mass. The blue dashed line is the effective range $r_{e}$, and the magenta dotted line is $r_{e, \text { Born }}=-\left(\mu / m_{A}\right) a_{\text {ho }}^{2} / a$, obtained in the Born approximation by replacing $\psi_{\text {reg }}(\rho)$ with $\psi_{0}(\rho, \rho)$

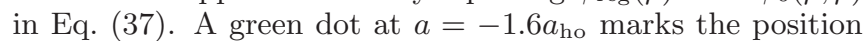
where $a_{\mathrm{eff}} \approx d$ if $V_{0}^{B}=50 E_{r}^{B}$.

of the external confinement. In the limit $k \rightarrow 0$, the resolvent has real matrix elements in position space, see Eq. (34); so does the symmetric operator $\hat{O}$, which then admits real eigenvalues $\lambda_{i}$ and orthonormal eigenvectors

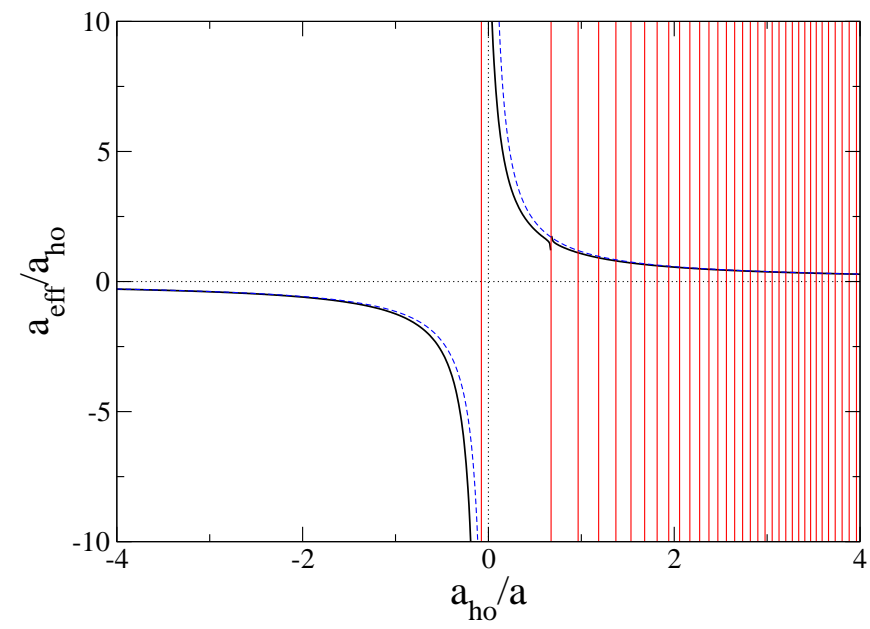

FIG. 11: (Color online) Same as Fig. 9 for $m_{B} / m_{A}=6.67$ (trapped ${ }^{40} \mathrm{~K}$ and free ${ }^{6} \mathrm{Li}$ ). The blue dashed line is the Born approximation $a_{\text {eff, Born }}=a m_{A} / \mu$, obtained by replacing $\psi_{\text {reg }}^{k=0}(\rho)$ by $\phi_{0}(\rho)$ in Eq. (38).

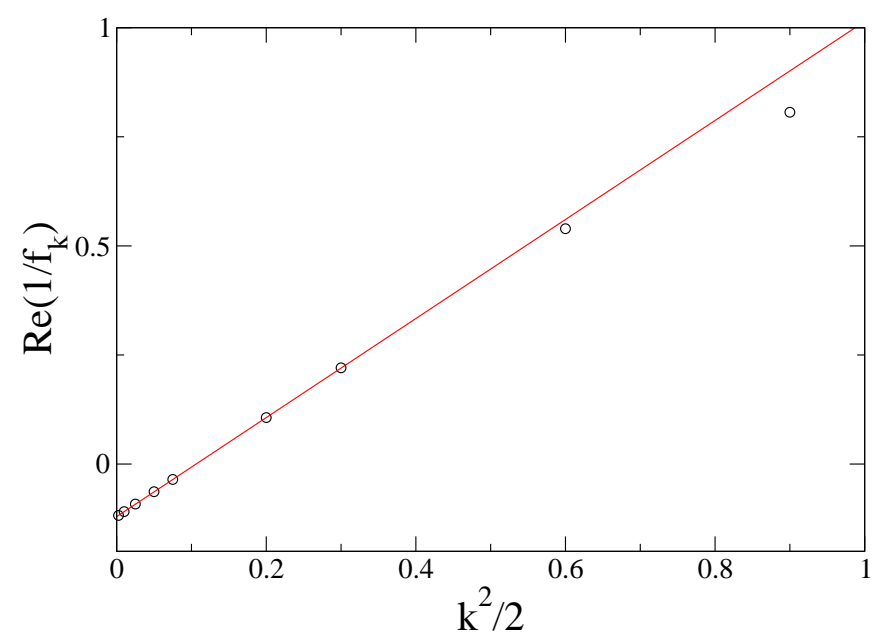

FIG. 12: (Color online) Energy dependence of the scattering amplitude for two particles of equal mass at $a=-1.6 a_{\mathrm{ho}}$ (green marker in Fig. 10). The linear fit at low energy, $\operatorname{Re}\left(1 / f_{k}\right)=-1 / a_{\mathrm{eff}}+r_{e} k^{2} / 2$, yields $a_{\mathrm{eff}}=8.3 a_{\mathrm{ho}}$ and $r_{e}=1.135 a_{\mathrm{ho}}$ (units of $a_{\mathrm{ho}}^{-2}$ and $a_{\mathrm{ho}}^{-1}$ on the horizontal and vertical axis, respectively).

$\left|e_{i}\right\rangle$ (see Eqs. E18E19). We then rewrite Eq. (38) as

$$
\begin{aligned}
a_{\mathrm{eff}} & =a \frac{m_{A}}{\mu}\left\langle\tilde{\psi}_{0}^{k=0}\left|\left(\mathrm{I}-g \hat{O}_{k=0}\right)^{-1}\right| \tilde{\psi}_{0}^{k=0}\right\rangle \\
& =\frac{m_{A}}{2 \pi \hbar^{2}} \sum_{i} \frac{\left|\left\langle\tilde{\psi}_{0}^{k=0} \mid \mathrm{e}_{i}\right\rangle\right|^{2}}{g^{-1}-\lambda_{i}}
\end{aligned}
$$

where the scalar product of functions of the single variable $R$ is defined as $\langle u \mid v\rangle=4 \pi \int_{0}^{+\infty} d R u^{*}(R) v(R)$. This means that a singularity in $a_{\text {eff }}$ is expected whenever the denominator of Eq. (42) vanishes, i.e. whenever the in- 
verse free space scattering length $1 / a$ equals $2 \pi \hbar^{2} \lambda_{i} / \mu$, provided that the non-interacting wave function has nonzero overlap with the corresponding eigenstate $\left|\mathrm{e}_{i}\right\rangle$.

Confinement-induced resonances have been been analyzed theoretically in different contexts, such as $1 \mathrm{D}$ wave guides [47, 48, 49], 3D optical lattices [50], and quasi-2D condensates [51]. In 1D wave guides the effect is particularly remarkable: due to the presence of the transverse confinement, a contact potential acquires a bound state for any value of the 3D scattering length (while in free space the contact potential has a bound state only for $a>0$ ). Confinement-induced modifications of two-body scattering properties have very recently been observed experimentally by the Zurich group in $1 \mathrm{D}$ waveguides 52 and in 3D optical lattices [53]. In most of the cited papers, the underlying translational symmetry and the harmonic nature of the confinement permit the factorization of the center-of-mass motion: this in turn implies that a single confinement-induced resonance can exist, since only one state in the closed channel is coupled to the open channel [48]. When this factorization is not possible, as in our setup or in the case of a 1D wave guide with anharmonic transverse confinement [9], an infinite set of states in the closed channel has non-zero coupling to the open one, and an infinite number of resonances appears. In practice however only a few of them may be resolved and relevant in an experiment since they become increasingly sharper as $a_{\mathrm{ho}} / a$ becomes larger (i.e. $\left|\left\langle\tilde{\psi}_{0} \mid \mathrm{e}_{i}\right\rangle\right|^{2} \rightarrow 0$ for large $i$ ). The position of these resonances can be predicted analytically in various limits, depending on the sign of $a$, as we now discuss.

\section{Position of the resonances for $a>0$}

When $a>0$, the pseudopotential admits a bound state in which the two particles can "sit" for a variable time, forming a molecule that oscillates in the harmonic well. To understand this point, we rewrite the two-body Hamiltonian (28) as

$$
\begin{array}{r}
-\frac{\hbar^{2} \Delta_{R}}{2\left(m_{A}+m_{B}\right)}+\frac{1}{2} m_{B} \omega^{2} R^{2}-\frac{\hbar^{2} \Delta_{r}}{2 \mu}+g \cdot \delta(\mathbf{r}) \cdot \frac{\partial}{\partial r}(r \cdot) \\
+\left[\frac{1}{2} \frac{m_{A} \mu}{m_{A}+m_{B}} \omega^{2} r^{2}-\mu \omega^{2} \mathbf{R} \cdot \mathbf{r}\right],
\end{array}
$$

and treat the terms in the square parenthesis as a perturbation. The unperturbed part admits the factorized eigenstates $\psi_{n, l=0}(\mathbf{R}, \mathbf{r})=\phi_{n, l=0}(\mathbf{R}) \chi(r)$ that describe a bound molecule with internal wavefunction $\chi(r)=$ $\exp (-r / a) / \sqrt{2 \pi a} r$ and center-of-mass in an eigenstate of the harmonic oscillator of angular momentum $l=0$ and radial quantum number $n \geq 0$. Since both the initial state and the Hamiltonian are spherically symmetric, conservation of angular momentum allows only $l=0$ intermediate molecular states. Within this unperturbed approximation, $a_{\text {eff }}$ diverges each time the energy of the oscillating molecule corresponds to the ground state energy of the pair of atoms, i.e. at the values of $a=a_{\text {res }}$ that satisfy

$$
\left(2 n+\frac{3}{2}\right) \hbar \omega \sqrt{\frac{m_{B}}{m_{A}+m_{B}}}-\frac{\hbar^{2}}{2 \mu a_{\mathrm{res}}^{2}}=\frac{3}{2} \hbar \omega .
$$

As can be seen in the upper part of Fig. 113 this formula describes the position of the resonances with $a>0$ in a wide region of the graph, since corrections to it are only $O\left(a / a_{\mathrm{ho}}\right)^{2}$ [54].

\section{Existence of a resonance for $a<0$}

For $a<0$ and for a large enough $m_{A} / m_{B}$ ratio, the presence of at least one resonance can be demonstrated by a very simple variational argument performed at the unitary limit $1 / a=0$. A contact potential characterized by the scattering length $a$ can be replaced by the simple boundary condition $\psi(\mathbf{R}, \mathbf{r}) \stackrel{r \rightarrow 0}{=} C(\mathbf{R})\left(r^{-1}-a^{-1}\right)+$ $o(1)$, where $C$ is an arbitrary function of the center of mass coordinate $\mathbf{R}$. The $s$-wave Ansatz

$$
\psi(R, r)=\mathcal{N} \exp \left(-R^{2} / \lambda^{2}\right) \frac{\exp \left(-r^{2} / \sigma^{2}\right)}{r},
$$

with $\lambda$ and $\sigma$ variational parameters, satisfies the boundary condition imposed by a unitarity limited contact potential, i.e. characterized by $1 / a=0$. Its energy can be calculated from the Hamiltonian (43): the term $\mathbf{R} \cdot \mathbf{r}$ has a vanishing contribution when averaged over this state, and the variational energy assumes the minimum value

$$
E^{\infty}=\frac{3}{2} \hbar \omega \sqrt{\frac{m_{B}}{m_{A}+m_{B}}}+\frac{1}{2} \hbar \omega \sqrt{\frac{m_{A}}{m_{A}+m_{B}}} .
$$

We might imagine continuously tuning $a$ from $0^{-}$, where no bound state can exist, towards $-\infty$. If $E^{\infty}<3 \hbar \omega / 2$ (i.e. if $m_{A} / m_{B}>9 / 16$ ) a bound state for $1 / a=0$ is guaranteed by the former Ansatz, and at least one resonance for $a_{\text {eff }}$ must exist in the $a<0$ region.

The following more elaborate calculation allows to prove the existence of a resonance for $a<0$, for an arbitrary mass ratio $m_{A} / m_{B}$. One performs a variational calculation directly on the integral operator $\hat{O}^{k=0}$, taking as a variational function $f(R)=R \exp \left(-R^{2} / 2 a_{\mathrm{ho}}^{2}\right)$. Using Eqs. E18 E19) and performing Gaussian integrals we obtain in harmonic oscillator units:

$$
\begin{aligned}
\left\langle f\left|\hat{O}^{k=0}\right| f\right\rangle= & \left(\frac{\alpha}{2}\right)^{3 / 2} \int_{0}^{+\infty} d \tau\left\{[(1+\alpha) \tau]^{-3 / 2}\right. \\
& \left.-[\tau+\alpha-\alpha \exp (-\tau)]^{-3 / 2}\right\} .
\end{aligned}
$$

From the inequality $\exp (-\tau)>1-\tau$ valid for any $\tau>0$, we conclude that the integrand is a negative function, so that $\left\langle f\left|\hat{O}^{k=0}\right| f\right\rangle<0$. Since the trial wavefunction $f(R)$ is proportional to $\tilde{\psi}_{0}^{k=0}(R)=R \phi_{0}(R)$, we conclude that 
$\hat{O}^{k=0}$ admits at least one eigenvector $\left|\mathrm{e}_{i}\right\rangle$ with a nonzero overlap with $\tilde{\psi}^{k=0}$ and with a negative eigenvalue $\lambda_{i}<0$. The identity Eq. (42) then implies the existence of a resonance in $a_{\text {eff for }} a<0$.

\section{Position of the resonances for $a<0$}

When $a<0$ and $m_{B} / m_{A} \ll 1$, the position of the resonances can be found with the aid of the BornOppenheimer approximation. For a fixed position of the massive particle $A$, one calculates the energy of the $B$ particle, which then constitutes an effective potential for the $A$ particle. Restricting for simplicity to the mean field regime $|a| \ll a_{\mathrm{ho}}$, one can assume that the particle $B$ remains in the ground state of the well, thereby creating an effective Gaussian attractive well for the $A$ particle. Hence the effective Hamiltonian for $A$ :

$$
H_{\mathrm{eff}}=-\frac{\hbar^{2}}{2 m_{A}} \Delta_{\mathbf{r}_{A}}-\frac{2 \pi \hbar|a|}{m_{B}} \frac{\exp \left(-r_{A}^{2} / a_{h o}^{2}\right)}{\left(\sqrt{\pi} a_{\mathrm{ho}}\right)^{3}}
$$

As argued above, the characteristic range of the potential is of order $a_{\text {ho }}$. This Hamiltonian can be easily solved numerically, and predicts a divergence of $a_{\text {eff }}$ whenever the combination $\left(|a| / a_{\mathrm{ho}}\right)\left(m_{A} / m_{B}\right)$ equals the critical value for the appearance of a new bound state (see Fig. 13 ] dashed lines in lower graph).

In the opposite limit $m_{B} / m_{A} \gg 1$, there is one resonance left on the $a<0$ side. It is intuitive that its position $a_{\text {res }}$ tends to $-\infty$ in this limit, the $B$ particle being then perceived by $A$ as a fixed scatterer of scattering length $a$, for which the resonance is obtained for $a=-\infty$. At $k=0$, one then expects that $\psi_{\text {reg }}(R) \propto \phi_{0}(R)$. To formalize this intuition, we expand the integral operator $\hat{O}$ in powers of the mass ratio $\alpha=m_{A} / m_{B} \rightarrow 0$ : from Eqs. E18E19, we get

$$
\hat{O}^{k=0}=\alpha^{3 / 2} \hat{O}_{0}+\alpha^{5 / 2} \hat{O}_{1}+\ldots
$$

It is then possible to check analytically, by calculation of Gaussian integrals, that one has exactly $\hat{O}_{0}\left|\tilde{\phi}_{0}\right\rangle=0$, with $\tilde{\phi}_{0}(R)=R \phi_{0}(R)$. Using perturbation theory, we obtain the series expansion of the lowest eigenvalue of $\hat{O}^{k=0}$ :

$$
\lambda_{0}=\alpha^{5 / 2}\left\langle\tilde{\phi}_{0}\left|\hat{O}_{1}\right| \tilde{\phi}_{0}\right\rangle+O\left(\alpha^{7 / 2}\right)=-\frac{\alpha^{5 / 2}}{\pi \sqrt{2}}+O\left(\alpha^{7 / 2}\right)
$$

in harmonic oscillator units. The resulting lowest order expression for the resonance position is

$$
\frac{a_{\mathrm{ho}}}{a_{\mathrm{res}}}=-\sqrt{2}\left(\frac{m_{A}}{m_{B}}\right)^{3 / 2}\left[1+O\left(\frac{m_{A}}{m_{B}}\right)\right],
$$

and is shown as a dashed line in Fig [13 [55].
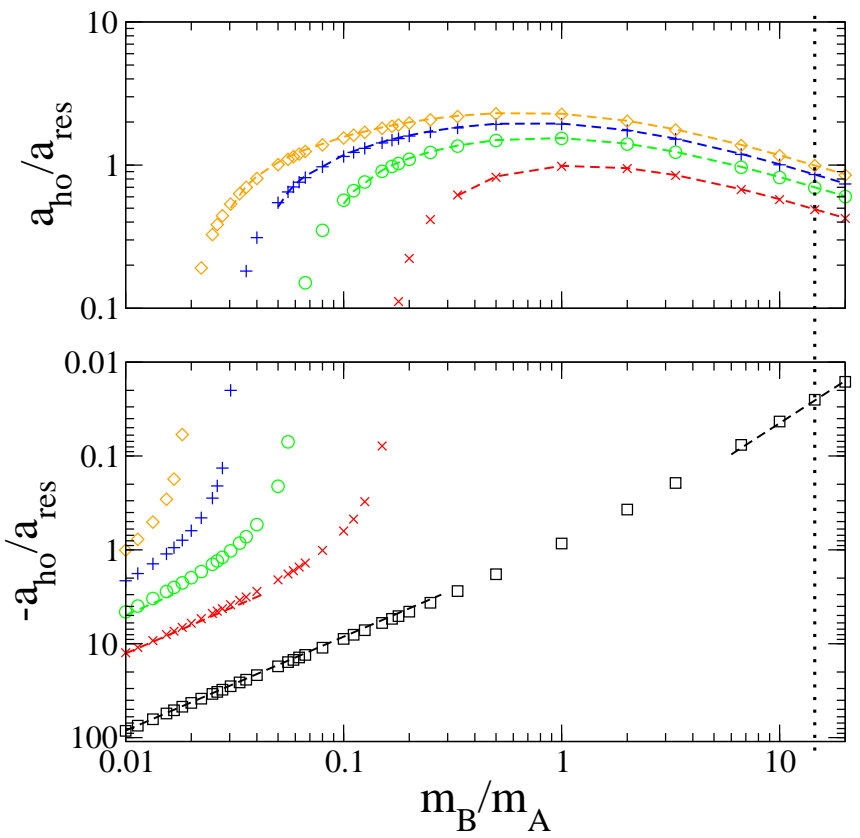

FIG. 13: (Color online) Position of the broadest resonances for positive (upper graph) and negative (lower graph) $a$ as a function of $m_{B} / m_{A}$. The dashed lines are the theoretical predictions: for $a>0$ they are given by Eq. (44); for $a<0$ and $m_{B}<m_{A}$ they are given by the Hamiltonian in Eq. 48); for $a<0$ and $m_{B}>m_{A}$ it is given by Eq. (51). In the upper graph, from top to bottom symbols correspond to $n=4,3,2,1$ in Eq. (44). The dotted vertical line indicates the value of the mass ratio $m_{B} / m_{A}$ when $A={ }^{6} \mathrm{Li}$ and $B={ }^{87} \mathrm{Rb}$.

\section{EXPERIMENTAL OUTLOOK AND CONCLUSIONS}

In this paper, we have shown numerically that the way of producing a disordered potential for matter waves proposed in 22], i.e. the use of atoms randomly trapped at the nodes of an optical lattice, indeed leads to the appearance of exponentially localized states in a threedimensional geometry. Our numerical method allows to directly compute the wavefunction of the localized states; it is based on the fact that the matrix elements of the resolvent of the Hamiltonian can be calculated extremely efficiently for the interaction of the matter wave with point scatterers, a fact already used with success in the context of light localization [23, 24, 42]. The method also allows to obtain analytical results in a straightforward manner, such as a check of the existence of localized states in 1D from Furstenberg theorem or the derivation of a link between the inverse lifetime of a localized state and its spatial decay in 3D, for a finite spatial extension of the scattering medium.

The main physical result is that numerous long-lived localized states appear for a wave number of the matter wave smaller than the inverse of the mean separation between scatterers, when the effective scattering length 
$a_{\text {eff }}$ of the matter wave on a trapped atom is positive and of the order of the mean separation of the scatterers. For the numerical examples of this paper, with a $10 \%$ occupancy of the lattice sites and for a wavenumber of the matter wave as large as $\sim 0.5 / d$, there are localized states with localization lengths that can be as small as the lattice period $d$, usually a sub-micron quantity. This extremely strong localization allows to have very long-lived localized states (lifetime larger that $10^{4}$ inverse recoil angular frequency of the matter wave) even in disordered samples with a radius as small as 10 lattice periods. By a full solution of the scattering problem of a free atom with a harmonically trapped one, we have shown that the required large values of $a_{\text {eff }} \sim d$ can be obtained by using an inter-species Feshbach resonance, and we have characterized intriguing confinement-induced resonances that appear in this two-body scattering process.

How to proceed in a real experiment to get evidence of these localized states ? A possibility is to extend to matter waves what was proposed for light in [24]: one introduces the matter wave wavepackets inside the scattering medium at a low value of $a_{\text {eff }}$, then one tunes $a_{\text {eff }}$ to the desired high value and one lets the matter wave evolve in presence of the scattering medium (but in the absence of an external trapping potential). After an adjustable time $\tau$, one measures the number of remaining matter wave atoms $N_{\text {rem }}(\tau)$ in the scattering medium. Since the component of the matter wave wavefunction in localized states decays exponentially in time with very weak rates $\Gamma$, the function $N_{\text {rem }}(\tau)$ should have a long tail, as compared to the case of a purely ballistic or even diffusive expansion [56]. A further check that this long tail has a decay rate varying exponentially with the size of the scattering medium would be a very convincing evidence of strong localization [58].

\section{Acknowledgments}

We are grateful to Uri Gavish, Dominique Delande, Martin Weitz, Leonardo Fallani, Chris. J. Pethick, Henrik Smith, Anders S. Sørensen, Fabrice Gerbier, Jean Dalibard and Christophe Salomon for stimulating discussions. Laboratoire Kastler Brossel is a research unit of École normale supérieure and of Université Pierre et Marie Curie, associated to CNRS. Our research group in Paris is a member of the IFRAF Institute.

\section{APPENDIX A: SPATIAL DECAY OF $\left\langle\mathbf{r}\left|\mathcal{G}\left(E+i 0^{+}\right)\right| \mathbf{r}^{\prime}\right\rangle$}

As illustrated in Fig. 14 in our model the off-diagonal matrix elements of the resolvent $\left\langle\mathbf{r}\left|\mathcal{G}\left(E+i 0^{+}\right)\right| \mathbf{r}^{\prime}\right\rangle$ decay exponentially in $\left|\mathbf{r}-\mathbf{r}^{\prime}\right|$ at low energy $E$. Is this a signature of localization? No, because this decay may be due to the fact that the energy $E$ is in a spectral gap of the system. And this occurs as well in the forbidden bands of a periodic system.

To illustrate this statement for our system, we have enclosed our lattice of scatterers in a box of side $L$, imposing periodic boundary conditions on the walls of the box. This amounts to replacing Eq. (10) by the particle propagator satisfying the correct boundary conditions:

$$
g_{0}^{\mathrm{Box}}(\mathbf{r})=\frac{2 m}{\hbar^{2} L^{3}} \sum_{\mathbf{q}} \frac{\mathrm{e}^{i \mathbf{q} \cdot \mathbf{r}}}{k^{2}-\mathbf{q}^{2}}
$$

with $\mathbf{q}=2 \pi \mathbf{n} / L$ and $\mathbf{n} \in \mathbb{Z}^{\mathbf{3}}$ (a triplet of integers). Indeed we found that, for the ensemble of scatterers used in Fig. [14 the ground state of the system, once enclosed in a box of side $L=23 d$ (slightly larger than the scattering medium), is characterized by a wave number $k_{\min }=0.7202 d^{-1}$. The exponential decay shown at $k=0.3 d^{-1}$ by $\left|\left\langle\mathbf{r}|\mathcal{G}| \mathbf{r}^{\prime}\right\rangle\right|^{2}$ is therefore simply indicating that at such low energy no state can exist deep inside the medium. In a scattering experiment, we might imagine a plane wave coming from infinity that scatters on the trapped $B$ atoms: if $k<k_{\min }$ the incoming wave undergoes total reflection, and inside the random medium only penetrates an evanescent wave, that decays exponentially from the boundary of the medium towards its interior. This example clearly points out that an exponential decay of $\left|\left\langle\mathbf{r}|\mathcal{G}| \mathbf{r}^{\prime}\right\rangle\right|^{2}$ is not a sufficient criterion to prove localization in our system, since it does not guarantee the existence of states deep inside the random potential.

In the criterion of Kramer and MacKinnon, introduced for a solid, the energy is taken equal to the Fermi energy, with the assumption that the density of states does not vanish at the Fermi energy. This criterion then turns out not to be practical in our case, since it requires a diagonalization of the Hamiltonian in order to calculate the density of states.

\section{APPENDIX B: ANALYTICAL RESULTS IN 1D}

We consider a 1D free space geometry with $N$ Dirac scatterers located in distinct positions $x_{1}<\ldots<x_{N}$. A quantum particle of mass $m$ interacts with the $N$ scatterers, with a coupling constant $g$. Using the transfer matrix formalism we calculate the resolvent $\mathcal{G}(z)$, for $z=E+i \eta$, $E$ real and $\eta>0$, which will give access to stationary wavefunctions in the limit $\eta \rightarrow 0^{+}$. Taking for simplicity $x_{0}=0$ different from the positions $x_{i}$, we use the fact that $\psi(x) \equiv\left\langle x|\mathcal{G}(z)| x_{0}=0\right\rangle$ solves Schrödinger's equation with a source term:

$$
\left[z+\frac{\hbar^{2}}{2 m} \frac{d^{2}}{d x^{2}}-g \sum_{i=1}^{N} \delta\left(x-x_{i}\right)\right] \psi(x)=\delta(x) .
$$

Over a position interval containing neither one of the $x_{i}$ nor $x_{0}=0, \psi(x)$ is an eigenstate of $d^{2} / d x^{2}$ so that, introducing the unique $k_{c}$ such that $z=\hbar^{2} k_{c}^{2} / 2 m$ with 


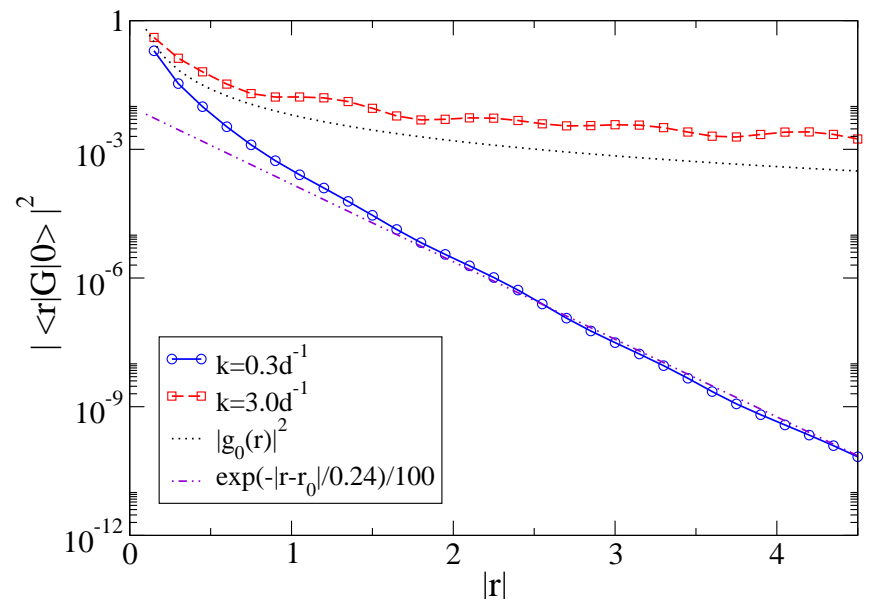

FIG. 14: (Color online) Decay of the off-diagonal real space matrix elements of the resolvent as a function of the distance from the center of the cloud of scatterers (in units of the lattice spacing $d$ ), averaged over 100 different realizations of the random potential. The $\approx 4600$ scatterers $\left(a_{\text {eff }}=0.3 d\right)$ are distributed in a cubic box of side $21 d$, occupying each site of the lattice with $p=0.5$. The y-axis is in units of $\left(\hbar^{2} / 2 m_{A}\right)^{-2}$.

$\operatorname{Im} k_{c}>0$, one has:

$$
\begin{array}{cll}
x \leq x_{1} & : & \psi(x)=A_{0}^{+} e^{i k_{c} x}+A_{0}^{-} e^{-i k_{c} x} \\
x_{j} \leq x \leq x_{j+1} & : \psi(x)=A_{j}^{+} e^{i k_{c} x}+A_{j}^{-} e^{-i k_{c} x} \\
x_{s} \leq x \leq 0 & : & \psi(x)=A_{s}^{+} e^{i k_{c} x}+A_{s}^{-} e^{-i k_{c} x} \\
0 \leq x \leq x_{s+1} & : & \psi(x)=B_{s}^{+} e^{i k_{c} x}+B_{s}^{-} e^{-i k_{c} x} \\
x_{j^{\prime}} \leq x \leq x_{j^{\prime}+1} & : & \psi(x)=B_{j^{\prime}}^{+} e^{i k_{c} x}+B_{j^{\prime}}^{-} e^{-i k_{c} x} \\
x_{N} \leq x & : \psi(x)=B_{N}^{+} e^{i k_{c} x}+B_{N}^{-} e^{-i k_{c} x}
\end{array}
$$

where we assumed that the first $s$ scatterer positions $x_{1}, \ldots, x_{s}$ are $<0$ and the $N-s$ other ones $x_{s+1}, \ldots, x_{N}$ are $>0$, and where $j$ runs from 1 to $s-1$ and $j^{\prime}$ from $s+1$ to $N-1$. All the unknown coefficients $A^{ \pm}$and $B^{ \pm}$shall now be determined from boundary conditions obeyed by $\psi(x)$.

A first boundary condition is that $\psi(x)$ should not diverge exponentially in $x= \pm \infty$. This imposes $A_{0}^{+}=0$ and $B_{N}^{-}=0$.

The other boundary conditions originate from the fact that each Dirac distribution in Eq. (B1) introduces a discontinuity of the first order derivative of $\psi(x)$, whereas $\psi(x)$ remains continuous. Integrating Eq. (B1) over an infinitesimal interval around $x_{j}$ leads to $\psi^{\prime}\left(x_{j}^{+}\right)-$ $\psi^{\prime}\left(x_{j}^{-}\right)=2 m g \psi\left(x_{j}\right) / \hbar^{2}$ with $\psi\left(x_{j}^{+}\right)=\psi\left(x_{j}^{-}\right)=\psi\left(x_{j}\right)$. These two equations allow to connect the unknown coefficients on the right of $x_{j}$ to the ones on the left by a two-by-two transfer matrix. For $j=1, \ldots, s$ we thus have

$$
\left(\begin{array}{c}
A_{j}^{+} \\
A_{j}^{-}
\end{array}\right)=P_{j}\left(\begin{array}{c}
A_{j-1}^{+} \\
A_{j-1}^{-}
\end{array}\right)
$$

and for $j=s+1, \ldots, N$ :

$$
\left(\begin{array}{c}
B_{j}^{+} \\
B_{j}^{-}
\end{array}\right)=P_{j}\left(\begin{array}{c}
B_{j-1}^{+} \\
B_{j-1}^{-}
\end{array}\right) .
$$

A simple calculation leads to the explicit expression

$$
P_{j}=\left(\begin{array}{cc}
1-i \gamma & -i \gamma e^{-2 i k_{c} x_{j}} \\
i \gamma e^{2 i k_{c} x_{j}} & 1+i \gamma
\end{array}\right)
$$

with $\gamma=m g /\left(\hbar^{2} k_{c}\right)$. We then introduce the two following matrices, one associated to the first $s$ scatterers,

$$
\mathcal{A} \equiv P_{s} \ldots P_{1}
$$

and the other one to the $N-s$ last scatterers:

$$
\mathcal{B} \equiv P_{s+1}^{-1} \ldots P_{N}^{-1} .
$$

They allow to express the coefficients $A_{s}^{ \pm}$in terms of $A_{0}^{-}$ and the coefficients $B_{s}^{ \pm}$in terms of $B_{N}^{+}$:

$$
\begin{aligned}
& \left(\begin{array}{c}
A_{s}^{+} \\
A_{s}^{-}
\end{array}\right)=\mathcal{A}\left(\begin{array}{c}
0 \\
A_{0}^{-}
\end{array}\right) \\
& \left(\begin{array}{c}
B_{s}^{+} \\
B_{s}^{-}
\end{array}\right)=\mathcal{B}\left(\begin{array}{c}
B_{N}^{+} \\
0
\end{array}\right) .
\end{aligned}
$$

The last two unknowns, $A_{0}^{-}$and $B_{N}^{+}$, are obtained from the boundary conditions imposed by $\delta(x)$ in Eq. (B1), $\psi\left(0^{-}\right)=\psi\left(0^{+}\right)$and $\psi^{\prime}\left(0^{+}\right)-\psi^{\prime}\left(0^{-}\right)=2 m / \hbar^{2}$, which imposes two equations on the coefficients $B_{s}^{ \pm}, A_{s}^{ \pm}$,

$$
\begin{aligned}
& A_{s}^{+}=B_{s}^{+}-\frac{m}{i \hbar^{2} k_{c}} \\
& A_{s}^{-}=B_{s}^{-}+\frac{m}{i \hbar^{2} k_{c}} .
\end{aligned}
$$

Combined with Eq. (B77) and Eq. (B8), this leads to the following system:

$$
\left(\begin{array}{ll}
\mathcal{B}_{11} & -\mathcal{A}_{12} \\
\mathcal{B}_{21} & -\mathcal{A}_{22}
\end{array}\right)\left(\begin{array}{c}
B_{N}^{+} \\
A_{0}^{-}
\end{array}\right)=\frac{m}{i \hbar^{2} k_{c}}\left(\begin{array}{r}
1 \\
-1
\end{array}\right),
$$

which can be solved explicitly:

$$
\begin{aligned}
A_{0}^{-} & =\frac{m}{i \hbar^{2} k_{c}} \frac{\mathcal{B}_{11}+\mathcal{B}_{21}}{\mathcal{B}_{11} \mathcal{A}_{22}-\mathcal{B}_{21} \mathcal{A}_{12}} \\
B_{N}^{+} & =\frac{m}{i \hbar^{2} k_{c}} \frac{\mathcal{A}_{12}+\mathcal{A}_{22}}{\mathcal{B}_{11} \mathcal{A}_{22}-\mathcal{B}_{21} \mathcal{A}_{12}} .
\end{aligned}
$$

We then proceed with the limit $z$ tending to a real and positive energy $E: \eta \rightarrow 0^{+}$and $k_{c} \rightarrow k=(2 m E)^{1 / 2} / \hbar$. The $P_{j}$ then become physically meaningful transfer matrices in the $S U(1,1)$ group. In the main text we introduced the reflection and transmission coefficients $r_{+}, t_{+}$ of the last $N-s$ first scatterers on the axis oriented from $x=-\infty$ to $x=+\infty$. This means that there exists a stationary solution of the usual Schrödinger equation equal 
to $e^{i k x}+r_{+} e^{-i k x}$ for $x<x_{s+1}$ and equal to $t_{+} e^{i k x}$ for $x>x_{N}$. By definition of the transfer matrices:

$$
\mathcal{B}\left(\begin{array}{c}
t_{+} \\
0
\end{array}\right)=\left(\begin{array}{c}
1 \\
r_{+}
\end{array}\right) \text {. }
$$

Similarly, introducing the reflection and transmission coefficients $r_{-}, t_{-}$of the first $s$ scatterers on the axis oriented this time from $x=+\infty$ to $x=-\infty$, we imply the existence of a solution of Schrödinger's equation equal to $e^{-i k x}+r_{-} e^{i k x}$ for $x>x_{s}$ and equal to $t_{-} e^{-i k x}$ for $x<x_{1}$, which imposes

$$
\mathcal{A}\left(\begin{array}{c}
0 \\
t_{-}
\end{array}\right)=\left(\begin{array}{c}
r_{-} \\
1
\end{array}\right) .
$$

This allows a physical interpretation of the coefficients of $\mathcal{A}$ and $\mathcal{B}$ :

$$
\begin{array}{ll}
\mathcal{A}_{12}=r_{-} / t_{-}, & \mathcal{A}_{22}=1 / t_{-} \\
\mathcal{B}_{21}=r_{+} / t_{+}, & \mathcal{B}_{11}=1 / t_{+} .
\end{array}
$$

This leads to very simple expressions for the Green's function $\psi(x)$ out of the scattering medium and over the interval between two scatterers containing $x_{0}=0$ :

$$
\begin{aligned}
x \leq x_{1}: \psi(x) & =\frac{m}{i \hbar^{2} k} \frac{t_{-}\left(1+r_{+}\right)}{1-r_{+} r_{-}} e^{-i k x} \\
x_{s} \leq x \leq 0: \psi(x) & =\frac{m}{i \hbar^{2} k} \frac{\left(1+r_{+}\right)\left(e^{-i k x}+r_{-} e^{i k x}\right)}{1-r_{+} r_{-}} \\
0 \leq x \leq x_{s+1}: \psi(x) & =\frac{m}{i \hbar^{2} k} \frac{\left(e^{i k x}+r_{+} e^{-i k x}\right)\left(1+r_{-}\right)}{1-r_{+} r_{-}} \\
x \geq x_{N}: \psi(x) & =\frac{m}{i \hbar^{2} k} \frac{t_{+}\left(1+r_{-}\right)}{1-r_{+} r_{-}} e^{+i k x} .
\end{aligned}
$$

\section{APPENDIX C: AN APPROXIMATE RELATION FOR SOME EXPECTATION VALUES OF Im $M$}

This appendix is useful to derive Eq. (22), an equation which relates the spatial decay of $\phi\left(\mathbf{r} ; \mathbf{r}_{0}\right)$ to the smallness of an eigenvalue of $M$.

Let us assume that we are at a positive energy $E$ such that the matrix $M$ has an eigenvalue $m_{0}$ extremely close to zero, much closer to zero anyway than all the other eigenvalues. Let $\overrightarrow{v_{0}}$ be the associated eigenvector of $M$. The corresponding adjoint vector is an eigenvector of $M^{\dagger}$ with the eigenvalue $m_{0}^{*}$. Since the matrix $M$ is (complex) symmetric, $M^{\dagger}$ is simply $M^{*}$, the complex conjugate of $M$, so that one may take as adjoint vector the complex conjugate ${\overrightarrow{v_{0}}}^{*}$ of $\overrightarrow{v_{0}}$. The imposed normalization condition is then

$$
\left\langle v_{0}^{*} \mid v_{0}\right\rangle={\overrightarrow{v_{0}}}^{2}=1
$$

For compactness we use here Dirac's notation, even if $M$ does not act in a Hilbert space.
One has to calculate the vector $\vec{d}$ to fully determine $\phi\left(\mathbf{r} ; \mathbf{r}_{0}\right)$, see Eq. (14). This vector solves the linear system

$$
M \vec{d}=\vec{s}
$$

with the source term $s_{j}=-\exp \left(i k\left|\mathbf{r}_{j}-\mathbf{r}_{0}\right|\right) /\left|\mathbf{r}_{j}-\mathbf{r}_{0}\right|$. As the eigenvalue $m_{0}$ of $M$ is the only one to be extremely close to zero, we take the approximate expression

$$
M^{-1} \simeq \frac{1}{m_{0}}\left|v_{0}\right\rangle\left\langle v_{0}^{*}\right| .
$$

This leads to

$$
\vec{d} \simeq \frac{1}{m_{0}} \overrightarrow{v_{0}}\left(\overrightarrow{v_{0}} \cdot \vec{s}\right) .
$$

To have access to an estimate of $\phi_{\text {out }}$, we have to calculate the expectation value of the imaginary part of the matrix $M$ on the vector $\vec{d}$. Since $M$ is symmetric, both the real part and the imaginary part of $M$ are hermitian matrices, with real expectation values, so that

$$
\vec{d}^{*} \cdot(\operatorname{Im} M) \vec{d}=\operatorname{Im}\left(\vec{d}^{*} \cdot M \vec{d}\right)=\operatorname{Im}\left(\vec{d}^{*} \cdot \vec{s}\right) .
$$

Then using the approximation Eq. (C4) we obtain

$$
\vec{d}^{*} \cdot(\operatorname{Im} M) \vec{d} \simeq \operatorname{Im}\left(\frac{\left({\overrightarrow{v_{0}}}^{*} \cdot \vec{s}^{*}\right)\left({\overrightarrow{v_{0}}}^{*} \cdot \vec{s}\right)}{m_{0}^{*}}\right) .
$$

An immediate application of this result is that the quantity $f(\mathbf{n})=\sum_{j} d_{j} e^{-i k \mathbf{n} \cdot \mathbf{r}_{j}}$, where $\mathbf{n}$ is a unity vector, is typically much larger than unity. The calculation of the average of $|f|^{2}$ over the unit sphere indeed leads to

$$
\left\langle|f|^{2}\right\rangle_{\mathbf{n}}=\frac{\vec{d}^{*} \cdot(\operatorname{Im} M) \vec{d}}{k}
$$

Since $|f| \gg 1$ in the low $m_{0}$ limit 63 , it is correct to neglect the term $e^{-i k \mathbf{n} \cdot \mathbf{r}_{0}}$ in Eq. (19) as was done in the main text.

To have access to an estimate of $\phi_{\text {in }}$, we have to calculate the maximal value of all the $\left|\operatorname{Im} d_{j}\right|$. Let us call $n$ the index such that $\left|\operatorname{Im} d_{n}\right|$ is the biggest one. According to the approximation Eq. (C4), we then have

$$
\operatorname{Im} d_{n} \simeq \operatorname{Im}\left[\frac{v_{0, n}\left(\overrightarrow{v_{0}} \cdot \vec{s}\right)}{m_{0}}\right]
$$

where $v_{0, n}$ denotes the component $n$ of the vector $\overrightarrow{v_{0}}$.

The expressions Eqs. C6 C8 greatly simplify when one chooses a position $\mathbf{r}_{0}$ that tends towards $\mathbf{r}_{n}$. The fact that the results shall not depend on this specific choice of $\mathbf{r}_{0}$ is established in the appendix D In this limit, all the components $s_{j}$ of the source term $\vec{s}$ are negligible as compared to $s_{n} \sim-1 /\left|\mathbf{r}_{0}-\mathbf{r}_{n}\right|$ so that $\overrightarrow{v_{0}} \cdot \vec{s} \sim-v_{0, n} / \mid \mathbf{r}_{0}-$ $\mathbf{r}_{n} \mid$ and so on. Then Eq. (21) reduces to

$$
\frac{\phi_{\text {in }}^{2}}{\phi_{\text {out }}^{2}} \simeq \frac{k R^{2}}{l^{2}} \operatorname{Im}\left[\frac{v_{0, n}^{* 2}}{m_{0}^{*}}\right] \text {. }
$$


As shown in the appendix $\mathbb{D}$ the components of the vector $\overrightarrow{v_{0}}$ are real in the limit of a vanishing $\left|m_{0}\right|$ so that $v_{0, n}^{2}$ may be pulled out of the imaginary part. Since the $\left|v_{0, j}\right|^{2}$ decrease roughly exponentially at large distances $\left|\mathbf{r}_{j}-\mathbf{r}_{n}\right|$ over a length scale $b$ of the order of the mean scatterer separation, as was known from studies of light localization [23, 24, 42], the normalization condition Eq. (C1) leads to $v_{0, n}^{2} \sim 1 /\left(\rho b^{3}\right) \lesssim 1$, where $\rho$ is the mean scatterers density. We then get Eq. (22).

\section{APPENDIX D: ON THE FACT THAT SOME QUANTITIES ARE ALMOST REAL}

We consider here a value of $k$ such that the matrix $M$ has one (and only one) eigenvalue $m_{0}$ of extremely small modulus. We then show that the corresponding eigenvector $\overrightarrow{v_{0}}$ of $M$, normalized as in Eq. (C1), is close to a vector with real components, a property used in the Appendix $[\mathrm{C}$ and necessary to obtain the equation Eq. (22).

First we give a physical argument. Starting from the approximation Eq. [C3], and keeping terms only to leading order in $1 / m_{0}$, we obtain from Eq. (14):

$$
\phi\left(\mathbf{r} ; \mathbf{r}_{0}\right) \simeq-A \operatorname{Im}\left[\frac{F(\mathbf{r}) F\left(\mathbf{r}_{0}\right)}{m_{0}}\right]
$$

where

$$
F(\mathbf{r})=\sum_{j=1}^{N} v_{0, j} \frac{e^{i k\left|\mathbf{r}-\mathbf{r}_{j}\right|}}{\left|\mathbf{r}-\mathbf{r}_{j}\right|} .
$$

Now let us assume that the phase of $F\left(\mathbf{r}_{0}\right)$ (modulo $\pi$ ) changes significantly (that is in a way not tending to zero with $m_{0}$ ) when $\mathbf{r}_{0}$ is varied. This means that the $\mathbf{r}$ dependent wavefunction $\phi\left(\mathbf{r} ; \mathbf{r}_{0}\right)$ spans a subspace of the Hilbert space of dimension 2 when $\mathbf{r}_{0}$ is varied, a subspace generated by the wavefunctions $\operatorname{Re} F(\mathbf{r})$ and $\operatorname{Im} F(\mathbf{r})$, or equivalently by $F(\mathbf{r})$ and $F^{*}(\mathbf{r})$. This leads to the physically suspect situation that two independent localized states can be associated to a given resonance.

To avoid this suspect situation, one is led to the assumption that the function $F(\mathbf{r})$ has a constant phase (modulo $\pi$ ). Taking the limit $\mathbf{r} \rightarrow \mathbf{r}_{j}$, this implies that all the $v_{0, j}$ have the same phase (modulo $\pi$ ). From the normalization condition Eq. (C1) we conclude that all the $v_{0, j}$ are real, apart from small terms tending to zero with $m_{0}$. Furthermore, this leads to the conclusion that the function $F(\mathbf{r})$ has to be real everywhere, apart from terms that tend to zero with $m_{0}$ 64. A consequence that is important for the appendix $\mathbb{C}$ is that $\overrightarrow{v_{0}}{ }^{*} \cdot \vec{s} \simeq{\overrightarrow{v_{0}}}^{*} \cdot \vec{s}^{*}=-F\left(\mathbf{r}_{0}\right)^{*}$ are approximately real, so that the result Eq. [C9] does not depend indeed on the specific choice of $\mathbf{r}_{0}$.

Now we give a mathematical argument to show that $\overrightarrow{v_{0}}$ is almost real. We start from $M \overrightarrow{v_{0}}=m_{0} \overrightarrow{v_{0}}$ and we split the matrix $M$ in a real part and an imaginary part,
$M=M_{R}+i M_{I}$. Since $M$ is symmetric, both $M_{R}$ and $M_{I}$ are real symmetric. Taking the squared norm of the identity $M_{R} \overrightarrow{v_{0}}=\left(m_{0} I-i M_{I}\right) \overrightarrow{v_{0}}$, where $I$ is the $N \times N$ identity matrix, leads to

$$
\left\|M_{R} \overrightarrow{v_{0}}\right\|^{2}={\overrightarrow{v_{0}}}^{*} \cdot\left[M_{I}^{2}+\operatorname{Re}\left(m_{0}^{2}\right) I\right] \overrightarrow{v_{0}},
$$

where we used the fact that

$$
{\overrightarrow{v_{0}}}^{*} \cdot M_{I} \overrightarrow{v_{0}}=\operatorname{Im}\left(m_{0}\right) \overrightarrow{v_{0}} * \overrightarrow{v_{0}}
$$

that can be proved as in Eq. C5. Next, we expand $\overrightarrow{v_{0}}$ in the orthonormal eigenbasis of $M_{I}, \overrightarrow{v_{0}}=\sum_{\alpha} c_{\alpha}\left|m_{I, \alpha}\right\rangle$, so that

$$
{\overrightarrow{v_{0}}}^{*} \cdot M_{I}^{2} \overrightarrow{v_{0}}=\sum_{\alpha}\left|c_{\alpha}\right|^{2} m_{I, \alpha}^{2}
$$

It remains to use the following property of $M_{I}$, valid for an arbitrary vector $\vec{x}$ :

$$
\vec{x}^{*} \cdot M_{I} \vec{x}=k \int \frac{d^{2} \mathbf{n}}{4 \pi}\left|\sum_{j} x_{j} e^{i k \mathbf{n} \cdot \mathbf{r}_{j}}\right|^{2}
$$

to show that $0 \leq m_{I, \alpha} \leq N k$ 65]. As a consequence, $m_{I, \alpha}^{2} \leq N k m_{I, \alpha}$. Using the expansion Eq. (D5) and Eq. (D4), one gets an upper bound on $\overrightarrow{v_{0}}{ }^{*} \cdot M_{I}^{2} \overrightarrow{v_{0}}$ so that

$$
\left\|M_{R} \overrightarrow{v_{0}}\right\|^{2} \leq\left[N k \operatorname{Im} m_{0}+\operatorname{Re}\left(m_{0}^{2}\right)\right]\left\|\overrightarrow{v_{0}}\right\|^{2} .
$$

In the large scattering medium diameter $L \rightarrow+\infty$, an exponential decrease of the imaginary part of $m_{0}$ is expected, $\sim \exp (-L / \xi)$. Close to the center of a resonance peak, $\left|\operatorname{Re} m_{0}\right| \sim \operatorname{Im} m_{0}$, so that $\left\|M_{R} \overrightarrow{v_{0}}\right\| /\left\|\overrightarrow{v_{0}}\right\|=$ $O[N k \exp (-L / 2 \xi)]$. On the contrary, the density of states for the spectrum of $M_{R}$ is not expected to be exponentially peaked: the spacing between successive eigenvalues is expected to scale as $1 / N$ at most. This is apparent in Fig 5 and we have checked it numerically for increasing numbers of scattering centers. This implies that $\overrightarrow{v_{0}}$, when expanded on the eigenvectors of $M_{R}$, populates essentially one eigenvector of $M_{R}$, the one with the eigenvalue nearest to zero. Since this (unit norm) eigenvector of $M_{R}$ is proportional to a real eigenvector, we deduce from Eq. C1 that the components of $\overrightarrow{v_{0}}$ are almost real, at the $O\left(\operatorname{Im} m_{0}\right)^{1 / 2}$ accuracy level.

\section{APPENDIX E: INTEGRAL EQUATION FOR $\psi_{\text {reg }}$}

To calculate the non-interacting two-particle Green's function numerically, we adapt a technique used in [66]. The Feynman propagator $K$ associated with the noninteracting Hamiltonian $H_{0}$ [67],

$$
K_{t}\left(\mathbf{r}_{A}, \mathbf{r}_{B} ; \boldsymbol{\rho}_{A}, \boldsymbol{\rho}_{B}\right)=\left\langle\mathbf{r}_{A}, \mathbf{r}_{B}\left|e^{-i H_{0} t / \hbar}\right| \boldsymbol{\rho}_{A}, \boldsymbol{\rho}_{B}\right\rangle
$$


can be factorized as the product of the two factors, $K_{t}=$ $K_{t}^{A} K_{t}^{B}$, the first term describing a free particle,

$K_{t}^{A}\left(\mathbf{r}_{A} ; \boldsymbol{\rho}_{A}\right)=e^{-i \pi 3 / 4}\left(\frac{m_{A}}{2 \pi \hbar t}\right)^{3 / 2} \exp \left(\frac{i m_{A}}{2 \hbar} \frac{\left|\mathbf{r}_{A}-\boldsymbol{\rho}_{A}\right|^{2}}{t}\right)$

and the second one a particle in a 3D harmonic oscillator,

$$
\begin{aligned}
& K_{t}^{B}\left(\mathbf{r}_{B} ; \boldsymbol{\rho}_{B}\right)=\phi(t) e^{-i \pi 3 / 4}\left(\frac{m_{B} \omega}{2 \pi \hbar|\sin (\omega t)|}\right)^{3 / 2} \\
& \exp \left(\frac{i m_{B} \omega}{\hbar}\left[\frac{r_{B}^{2}+\rho_{B}^{2}}{2 \tan (\omega t)}-\frac{\mathbf{r}_{B} \cdot \boldsymbol{\rho}_{B}}{\sin (\omega t)}\right]\right)
\end{aligned}
$$

where $\phi(t)=\exp (i \pi n / 2)$ for $n \pi<\omega t<(n+1) \pi$. Setting $K_{t}=0$ for $t<0, G_{E}$ is obtained as the Fourier transform of $K_{t}$,

$$
G_{E}=-\frac{i}{\hbar} \int_{0}^{\infty} d t e^{i\left(E+i 0^{+}\right) t / \hbar} K_{t}
$$

For simplicity, we introduce dimensionless variables by expressing quantities in harmonic oscillator units and, even though the derivation has been carried out for a generic mass ratio, we restrict ourselves here to the special case $m_{A}=m_{B}=m$ (see end of appendix for the case $k \rightarrow 0$ with an arbitrary mass ratio $\left.m_{A} / m_{B}\right)$.

In order to find the equation satisfied by $\psi_{\text {reg, }}$ we rewrite Eq. (30) as

$$
\begin{aligned}
& \psi\left(\mathbf{r}_{A}, \mathbf{r}_{B}\right)=\psi_{0}\left(\mathbf{r}_{A}, \mathbf{r}_{B}\right)+g \psi_{\mathrm{reg}}(R) \int d \boldsymbol{\rho} G_{E}\left(\mathbf{r}_{A}, \mathbf{r}_{B} ; \boldsymbol{\rho}, \boldsymbol{\rho}\right) \\
& \quad+g \int d \boldsymbol{\rho} G_{E}\left(\mathbf{r}_{A}, \mathbf{r}_{B} ; \boldsymbol{\rho}, \boldsymbol{\rho}\right)\left[\psi_{\mathrm{reg}}(\rho)-\psi_{\mathrm{reg}}(R)\right]
\end{aligned}
$$

and let the two particles approach each other. We introduce here the center-of-mass and relative coordinates $\mathbf{R}=\left(\mathbf{r}_{A}+\mathbf{r}_{B}\right) / 2$ and $\mathbf{r}=\mathbf{r}_{A}-\mathbf{r}_{B}$. Let us turn our attention to the first integral appearing in Eq. (E5):

$$
U \equiv \int d \boldsymbol{\rho} G_{E}\left(\mathbf{R}+\frac{\mathbf{r}}{2}, \mathbf{R}-\frac{\mathbf{r}}{2} ; \boldsymbol{\rho}, \boldsymbol{\rho}\right)
$$

Performing the Gaussian integration over $\boldsymbol{\rho}$ [68] one finds

$$
U=\frac{e^{i \pi 3 / 4}}{(2 \pi)^{3 / 2}} \int_{0}^{\infty} d t \frac{\tilde{\phi}(t) e^{i(E t+W)}}{|\sin (t)+t \cos (t)|^{3 / 2}}
$$

where

$$
W=\frac{\left|\mathbf{R}+\frac{\mathbf{r}}{2}\right|^{2}}{2 t}+\frac{\left|\mathbf{R}-\frac{\mathbf{r}}{2}\right|^{2}}{2 \tan (t)}-\frac{1}{x}\left|\frac{\mathbf{R}+\frac{\mathbf{r}}{2}}{2 t}+\frac{\mathbf{R}-\frac{\mathbf{r}}{2}}{2 \sin (t)}\right|^{2} .
$$

We have here introduced the shorthand notation $x(t)=$ $[1 / t+1 / \tan (t)] / 2$ and the phase factor $\tilde{\phi}(t)$, which equals $\exp (i \pi n / 2)$ for $t_{n}<t<t_{n+1}$, where $t_{0}=0$ and $t_{1}=2.029, t_{2}=4.913, \ldots$ are the consecutive solutions of $x(t)=0$. In the latter expression, Eq. (E7), the contribution of the neighborhood of $t=0$ diverges as $1 / r$ for $r \rightarrow 0$

$$
\begin{aligned}
\frac{(2 \pi)^{3 / 2}}{e^{i \pi 3 / 4}} U & =\int_{0}^{\delta} d t \frac{e^{i r^{2} / 4 t}}{(2 t)^{3 / 2}}+\int_{\delta}^{\infty} d t \ldots \\
& =\int_{0}^{\infty} d t \frac{e^{i r^{2} / 4 t}}{(2 t)^{3 / 2}}+\int_{\delta}^{\infty} d t\left(-\frac{1}{(2 t)^{3 / 2}}+\ldots\right) \\
& =\frac{1}{r} \sqrt{\frac{\pi}{2}} e^{i \pi / 4}+\int_{\delta}^{\infty} d t\left(-\frac{1}{(2 t)^{3 / 2}}+\ldots\right)
\end{aligned}
$$

We finally find

$$
\begin{aligned}
g \psi_{\mathrm{reg}}(R) \int & d \boldsymbol{\rho} G_{E}\left(\mathbf{R}+\frac{\mathbf{r}}{2}, \mathbf{R}-\frac{\mathbf{r}}{2} ; \boldsymbol{\rho}, \boldsymbol{\rho}\right) \\
& \stackrel{r \rightarrow 0}{=} \psi_{\mathrm{reg}}(R)\left(g F_{1}(R)-\frac{a}{r}\right)+o(1)
\end{aligned}
$$

that separates out the expected $1 / r$ divergent contribution and defines

$F_{1}(R)=\frac{e^{i \pi 3 / 4}}{(2 \pi)^{3 / 2}} \int_{0}^{\infty} \mathrm{d} t\left(\frac{\tilde{\phi}(t) e^{i\left(E t+W_{0}\right)}}{|\sin (t)+t \cos (t)|^{3 / 2}}-\frac{1}{(2 t)^{3 / 2}}\right)$

with $W_{0}=R^{2}(\cos (t)-t \sin (t) / 2-1) /(\sin (t)+t \cos (t))$. The term $(2 t)^{-3 / 2}$ regularizes $F_{1}$ in the neighborhood of $t=0$, and we have taken the limit $\delta \rightarrow 0$.

Let us now consider the remaining term appearing in Eq. E5): when $\mathbf{r} \rightarrow 0$ and $\mathbf{u}=\mathbf{R}-\boldsymbol{\rho} \rightarrow 0$, the Green's function $G_{E}$ diverges as $|\mathbf{u}|^{-4}$, or equivalently as $\left(\left|\mathbf{r}_{A}-\boldsymbol{\rho}_{A}\right|^{2}+\left|\mathbf{r}_{B}-\boldsymbol{\rho}_{B}\right|^{2}\right)^{-2}$, but the second integral in Eq. E5 is convergent in $\mathbf{u} \sim 0$ :

$$
\begin{aligned}
& \int d \boldsymbol{\rho} G_{E}\left(\mathbf{R}+\frac{\mathbf{r}}{2}, \mathbf{R}-\frac{\mathbf{r}}{2} ; \boldsymbol{\rho}, \boldsymbol{\rho}\right)\left[\psi_{\text {reg }}(\rho)-\psi_{\text {reg }}(R)\right] \\
& \stackrel{\mathbf{r} \rightarrow 0}{=} \int \frac{d \mathbf{u}}{|\mathbf{u}|^{4}}\left[\left.\mathbf{u} \frac{\partial \psi_{\text {reg }}}{\partial \mathbf{u}}\right|_{\mathbf{u}=\mathbf{0}}+O\left(|\mathbf{u}|^{2}\right)\right](\mathrm{E} 12
\end{aligned}
$$

(the first order term vanishes due to spherical symmetry). In this term therefore no divergence arises and we may set $r=0$. The angular integrations can be performed analytically and we obtain:

$$
\begin{aligned}
\int & d \boldsymbol{\rho} G_{E}(\mathbf{R}, \mathbf{R} ; \boldsymbol{\rho}, \boldsymbol{\rho})\left[\psi_{\mathrm{reg}}(\rho)-\psi_{\mathrm{reg}}(R)\right] \\
= & \frac{1}{R} \int_{0}^{\infty} d \rho\left[\tilde{\psi}_{\mathrm{reg}}(\rho)-\frac{\rho}{R} \tilde{\psi}_{\mathrm{reg}}(R)\right] F_{2}(R, \rho)
\end{aligned}
$$

with

$$
F_{2}(R, \rho)=\int_{0}^{\infty} d t \frac{\phi(t) e^{i\left[E t+\left(R^{2}+\rho^{2}\right) x\right]}}{(t|\sin (t)|)^{3 / 2}} \frac{\sin (2 R \rho y)}{(2 \pi)^{2} y}
$$

which is symmetric under the exchange of $\rho$ and $R$. We have here introduced the radial wave function $\tilde{\psi}(R)=$ $R \psi(R)$ and the function $y(t)=[1 / t+1 / \sin (t)] / 2$. 


\section{Writing}

$$
\psi\left(\mathbf{r}_{A}, \mathbf{r}_{B}\right) \stackrel{r \rightarrow 0}{=} \psi_{\mathrm{reg}}(R)\left(1-\frac{a}{r}\right)+o(1),
$$

we can cancel the divergent contribution on both sides of Eq. (E5), and the remaining terms constitute the implicit integral equation

$$
\begin{aligned}
& \tilde{\psi}_{\mathrm{reg}}(R)=\tilde{\psi}_{0}(R, R)+g F_{1}(R) \tilde{\psi}_{\mathrm{reg}}(R) \\
& \quad+g \int_{0}^{+\infty} d \rho F_{2}(R, \rho)\left[\tilde{\psi}_{\mathrm{reg}}(\rho)-\frac{\rho}{R} \tilde{\psi}_{\mathrm{reg}}(R)\right]
\end{aligned}
$$

that needs to be solved numerically in order to determine $\psi_{\text {reg }}$. The latter equation can be written in a symbolic, more compact form as

$$
\tilde{\psi}_{\mathrm{reg}}=\frac{I}{I-g \hat{O}} \tilde{\psi}_{0}
$$

where $\hat{O}$ is a symmetric integral operator, which is real in the limit $k \rightarrow 0$.

If one only aims at calculating strictly zero energy properties (i.e. $a_{\mathrm{eff}}$, and not $f_{k}$ or $r_{e}$ ), the treatment presented in this Appendix can be drastically simplified setting $\tau=i t / \hbar$ in Eqs. (E2 E3E4) and $E=3 \hbar \omega / 2$ in Eq. (E4): the derivation of $\hat{O}$ proceeds in an analogous way, but the resulting integral equation is much easier to solve since the integrand in both $F_{1}$ and $F_{2}$ become real damped functions with no finite time singularities. For an arbitrary value of the mass ratio $\alpha \equiv m_{A} / m_{B}$, they are given by:

$$
\begin{aligned}
& F_{1}(R)=-\frac{1}{(2 \pi)^{3 / 2}} \int_{0}^{\infty} d \tau\left\{-\frac{1}{\left[\left(1+\frac{1}{\alpha}\right) \tau\right]^{3 / 2}}+\right. \\
& \left.\frac{\exp (3 \tau / 2)}{\left[\operatorname{sh}(\tau)+\frac{\tau}{\alpha} \operatorname{ch}(\tau)\right]^{3 / 2}} \exp \left(-R^{2} \frac{\operatorname{ch}(\tau)+\frac{\tau}{2 \alpha} \operatorname{sh}(\tau)-1}{\operatorname{sh}(\tau)+\frac{\tau}{\alpha} \operatorname{ch}(\tau)}\right)\right\},
\end{aligned}
$$

and

$$
F_{2}(R, \rho)=-\alpha^{3 / 2} \int_{0}^{\infty} d \tau \frac{\exp \left[-\left(R^{2}+\rho^{2}\right) x\right]}{[\tau \operatorname{sh}(\tau) \exp (-\tau)]^{3 / 2}} \frac{\operatorname{sh}(2 R \rho y)}{(2 \pi)^{2} y}
$$

with $x(\tau)=\alpha / 2 \tau+1 / 2 \operatorname{th}(\tau)$ and $y(\tau)=\alpha / 2 \tau+1 / 2 \operatorname{sh}(\tau)$.
[1] C. A. Regal, M. Greiner and D. S. Jin, Phys. Rev. Lett. 92, 040403 (2004); M.W. Zwierlein, C.A. Stan, C.H. Schunck, S. M. F. Raupach, A. J. Kerman, W. Ketterle, Phys. Rev. Lett. 92, 120403 (2004); M. Bartenstein, A. Altmeyer, S. Riedl, S. Jochim, C. Chin, J. H. Denschlag, R. Grimm, Phys. Rev. Lett. 92, 120401 (2004); T. Bourdel, L. Khaykovich, J Cubizolles, J. Zhang, F. Chevy, M. Teichmann, L. Tarruell, S.J.J.M.F. Kokkelmans, C. Salomon, Phys. Rev. Lett. 93, 050401 (2004); C. Chin, M. Bartenstein, A. Altmeyer, S. Riedl, S. Jochim, J. Hecker Denschlag, R. Grimm, Science 305, 1128 (2004); J. Kinast, A. Turlapov, J. E. Thomas, Q. Chen, J. Stajic, K. Levin, Science 307, 1296 (2005).

[2] M. Greiner, O. Mandel, T. Esslinger, T. W. Hänsch, and I. Bloch, Nature 415, 39 (2002).

[3] G. Montambaux and E. Akkermans, Physique mésoscopique des électrons et des photons (EDP Sciences, 2004).

[4] P. A. Lee and T. V. Ramakrishnan, Rev. Mod. Phys. 57, 287 (1985).

[5] B. Kramer and A. MacKinnon, Rep. Prog. Phys. 56, 1469 (1993).

[6] B. Damski, J. Zakrzewski, L. Santos, P. Zoller, and M. Lewenstein Phys. Rev. Lett. 91, 080403 (2003)

[7] A. Sanpera, A. Kantian, L. Sanchez-Palencia, J. Zakrzewski, and M. Lewenstein, Phys. Rev. Lett. 93, 040401 (2004).

[8] L. Fallani, J. E. Lye, V. Guarrera, C. Fort, M. Inguscio, cond-mat/0603655

[9] S. Ospelkaus, C. Ospelkaus, O. Wille, M. Succo, P. Ernst, K. Sengstock, K. Bongs, Phys. Rev. Lett. 96, 180403 (2006).
[10] J. von Neumann and E. Wigner, Phys. Z. 30, 465 (1929). This paper contains a superficial algebraic error, that has been corrected in: F. H. Stillinger and D. R. Herrick, Phys. Rev. A 11, 446 (1975).

[11] P. W. Anderson, Phys. Rev. 109, 1492 (1958).

[12] D. S. Wiersma, P. Bartolini, A. Lagendijk, and R. Righini, Nature 390, 671 (1997).

[13] T. Jonckheere, C. A. Mueller, R. Kaiser, C. Miniatura, D. Delande, Phys. Rev. Lett. 85, 4269 (2000); G. Labeyrie, D. Delande, C. A. Mueller, C. Miniatura, R. Kaiser, Europhys. Lett. 61, 327 (2003); G. Labeyrie, D. Delande, R. Kaiser, C. Miniatura, cond-mat/0603153

[14] J. M. Vogels, C. C. Tsai, R. S. Freeland, S. J. J. M. F. Kokkelmans, B. J. Verhaar, and D. J. Heinzen, Phys. Rev. A 56, R1067 (1997); S. Inouye, M.R. Andrews, J. Stenger, H.-J. Miesner, D.M. Stamper-Kurn, and W. Ketterle, Nature 392, 151-154 (1998).

[15] D. Boiron, C. Mennerat-Robilliard, J.-M. Fournier, L. Guidoni, C. Salomon, and G. Grynberg, Eur. Phys. J. D 7, 373 (1999).

[16] J. E. Lye, L. Fallani, M. Modugno, D.S. Wiersma, C. Fort, and M. Inguscio, Phys. Rev. Lett. 95, 070401 (2005).

[17] D. Clément, A. F. Varon, M. Hugbart, J. A. Retter, P. Bouyer, L. Sanchez-Palencia, D. M. Gangardt, G. V. Shlyapnikov, and A. Aspect, Phys. Rev. Lett. 95, 170409 (2005).

[18] C. Fort, L. Fallani, V. Guarrera, J. E. Lye, M. Modugno, D. S. Wiersma, and M. Inguscio, Phys. Rev. Lett. 95, 170410 (2005).

[19] T. Schulte, S. Drenkelforth, J. Kruse, W. Ertmer, J. Arlt, K. Sacha, J. Zakrzewski, and M. Lewenstein, Phys. Rev. 
Lett. 95, 170411 (2005)

[20] M. Modugno, Phys. Rev. A 73, 013606 (2006).

[21] R. C. Kuhn, C. Miniatura, D. Delande, O. Sigwarth, C. A. Müller, Phys. Rev. Lett. 95, 250403 (2005).

[22] U. Gavish and Y. Castin, Phys. Rev. Lett. 95, 020401 (2005).

[23] M. Rusek, A. Orlowski, Phys. Rev. E 51, R2763 (1995).

[24] Emmanuel Mandonnet, PhD Thesis of Université Paris 6 (2000), online at http://tel.ccsd.cnrs.fr/tel-00011872

[25] S. E. Hamann, D. L. Haycock, G. Klose, P. H. Pax, I. H. Deutsch, and P. S. Jessen, Phys. Rev. Lett. 80, 4149 (1998).

[26] H. Perrin, A. Kuhn, I. Bouchoule, and C. Salomon, Europhys. Lett. 42, 395 (1998).

[27] We used the asymptotic formula for the half-width of the ground energy band in a 1D standing wave of modulation depth $V_{0}^{B}: \quad \delta E_{0}=\hbar / t_{\text {tunnel }} \simeq$ $(8 / \sqrt{\pi})\left(V_{0}^{B}\right)^{3 / 4}\left(E_{r}^{B}\right)^{1 / 4} \exp \left[-2\left(V_{0}^{B} / E_{r}^{B}\right)^{1 / 2}\right]$, a formula taken from R. Campbell, Théorie générale de l'équation de Mathieu et de quelques autres équations différentielles de la Mécanique (Masson, 1955). With this definition of the tunneling time, the probability for the $B$ particle not to have tunneled during $t$ behaves as $\exp \left(-D t^{2} / t_{\text {tunnel }}^{2}\right)$ at short times, where $D=3$ is the spatial dimension of the lattice.

[28] If $A$ is a gas of fermionic ${ }^{40} \mathrm{~K}$ atoms $\left(\lambda_{A}=770 \mathrm{~nm}\right)$ and $B$ a gas of ${ }^{87} \mathrm{Rb}$, the calculation for $\lambda_{L}=779 \mathrm{~nm}$ yields $V_{0}^{A}=-3.6 E_{r}^{A}$. Due to the smaller detuning and the larger mass ratio $m_{A} / m_{B}$, the optical potential would induce noticeable effects on the potassium matter wave.

[29] D. Petrov, Phys. Rev. A 67, 010703(R) (2003).

[30] Eqs. $(11,12)$ of [29] are derived under the condition that the kinetic energy of the three particles in their centerof-mass frame is less than the dimer binding energy $\epsilon$ :

$\frac{\hbar^{2}\left(k_{1}^{2}+k_{2}^{2}\right)}{2 m_{A}}+\frac{\hbar^{2} k_{3}^{2}}{2 m_{B}}-\frac{\hbar^{2}\left(\overrightarrow{k_{1}}+\overrightarrow{k_{2}}+\overrightarrow{k_{3}}\right)^{2}}{2\left(m_{B}+2 m_{A}\right)} \lesssim \frac{\hbar^{2}}{2 \mu a_{\text {eff }}^{2}}$

where $\mu^{-1}=m_{A}^{-1}+m_{B}^{-1}, \vec{k}_{1}, \vec{k}_{2}$ are the wavevectors of the two $A$ atoms and $\overrightarrow{k_{3}}$ is the wavevector of the $B$ atom. Whereas low enough values of $k_{1}, k_{2}<k_{F}$ may be chosen by adjusting the density of $A$ atoms, the value of $k_{3}$ is fixed by the zero-energy oscillation of the trapped $B$ particle, $\hbar^{2} k_{3}^{2} / 2 m_{B} \simeq \hbar \omega$. The $k_{1}=k_{2}=0$ limit of Eq. E20 therefore results in a condition that looks difficult to satisfy in general for $a_{\text {eff }} \sim d$ :

$$
\hbar \omega \frac{2 m_{A}}{m_{B}+2 m_{A}} \lesssim \frac{\hbar^{2}}{2 \mu a_{\text {eff }}^{2}} .
$$

In the limit $m_{A} \ll m_{B}$, however, as it is the case for the Li-Rb mixture, the vibrational energy in Eq. E21 is multiplied by a small number $\sim 2 m_{A} / m_{B}$ whereas the binding energy $\epsilon$ increases as $1 / m_{A}$ : for $a_{\text {eff }}=d=$ $\pi / k_{L}$ and $V_{0}^{B}=50 E_{r}^{B}$, we thus find that the condition is satisfied for $A={ }^{6} \mathrm{Li}, B={ }^{87} \mathrm{Rb}$, so that Eqs. $(11,12)$ of [29] may be used.

[31] The $T$ matrix of the matter wave is related to $M$ by $T\left(E+i 0^{+}\right)=\left(2 \pi \hbar^{2} / m_{A}\right) \sum_{j, l}\left[M^{-1}\right]_{j l}\left|\mathbf{r}_{j}\right\rangle\left\langle\mathbf{r}_{l}\right|$.

[32] J. M. Ziman, Models of Disorder (Cambridge University Press, Cambridge, England, 1979), chapter 8.

[33] This results from the fact that the Hamiltonian $\mathcal{H}$ is real in position space. Let $|\psi\rangle \equiv \mathcal{G}(E+i \eta)\left|\mathbf{r}_{0}\right\rangle$. By definition of $\mathcal{G}$,

$$
(E+i \eta-\mathcal{H}) \psi(\mathbf{r})=\delta\left(\mathbf{r}-\mathbf{r}_{0}\right),
$$

where $\mathcal{H}$ is a real differential operator. Taking the limit $\eta \rightarrow 0^{+}$of the imaginary part of the latter equation leads to $\mathcal{H} \phi=E \phi$. Alternatively, using the spectral expansion of the Hamiltonian and of the resolvent with real eigenwavefunctions, one finds $\phi\left(\mathbf{r} ; \mathbf{r}_{0}\right)=-\pi\left\langle\mathbf{r}\left|P_{E}\right| \mathbf{r}_{0}\right\rangle$, where $P_{E}$ is the projector on the eigenspace of $\mathcal{H}$ of energy $E$. This shows that, when one takes all possible values of $\mathbf{r}_{\mathbf{0}}$, the wavefunctions $\phi$ span the entire eigenspace of energy $E>0$. For $E=0$, the density of states vanishes in 3D, and so does $\phi$.

[34] H. Furstenberg, Trans. Am. Math. Soc. 108, 377 (1963); for the application of this theorem to a product of transfer matrices, see K. Ishii, Supplement of the Progress of Theoretical Physics, 53, 77 (1973), and V. Baluni, J. Willemsen, Phys. Rev. A 31, 3358 (1985).

[35] This does not hold, however, when $r_{+}$or $r_{-}$is a real negative number, in which case one recovers the generic behavior of $\phi(x)$ depicted in Fig 2 . This means that $x_{0}=$ 0 is a 'bad' choice, corresponding to the location of a node of the localized state.

[36] We define $\phi_{\mathrm{in}}^{2}$ as the average of the 6 values $\phi^{2}\left(\mathbf{r}_{\text {in }} ; \mathbf{r}_{\text {in }}\right)$, with $\mathbf{r}_{\text {in }}$ chosen between the 6 values $\{(d / 2,0,0),(-d / 2,0,0)$, and cyclic permutations $\}$. Similarly, we define $\phi_{\text {out }}^{2}$ as the average of the 36 values $\phi^{2}\left(\mathbf{r}_{\text {out }} ; \mathbf{r}_{\text {in }}\right)$, with $\mathbf{r}_{\text {out }}$ chosen between the 6 values $\{(20 d, 0,0),(-20 d, 0,0)$, and cyclic permutations $\}$, and $\mathbf{r}_{\text {in }}$ as before.

[37] Requiring that the modulus square of the wavefunction decreases by four orders of magnitude over the radius of the medium (which is a minimal requirement to pretend to be localized) yields for the parameters of Fig. 4a maximal localization length of the order of $4 d$. The numerical observation of larger values of $\xi$ would require a much larger scattering medium.

[38] A. Shalgi and Y. Imry, Les Houches Session LXI, Mesoscopic Quantum Physics (E. Akkermans, G. Montambaux, J.-L. Pichard and J. Zinn-Justin eds.), 1995.

[39] A strong reformulation of this far field extrapolation technique is to note that, out of a sphere containing the scattering medium, the function $\chi(\mathbf{r})=\sum_{j} d_{j} \exp (i k \mid \mathbf{r}-$ $\left.\mathbf{r}_{j} \mid\right) /\left|\mathbf{r}-\mathbf{r}_{j}\right|$ is an eigenstate of the Laplacian with the eigenvalue $-k^{2}$ so that it admits the multipolar expansion on spherical harmonics, $\chi(\mathbf{r})=\sum_{l, m} c_{l, m} Y_{l}^{m}(\theta, \phi) f_{l}(r)$, where $f_{l}(r)$ solves the spherical Bessel differential equation with the asymptotic behavior at large $r, f_{l}(r) \sim$ $\exp (i k r) / r$. The complex coefficients $c_{l, m}$ obey the exact relation:

$$
\sum_{l, m}\left|c_{l, m}\right|^{2}=\frac{4 \pi}{k} \vec{d}^{*} \cdot \operatorname{Im} M \vec{d} \propto R^{2} \phi_{\text {out }}^{2}
$$

where $\phi_{\text {out }}$ is obtained from the far field extrapolation. So showing that this $\phi_{\text {out }}$ is small amounts to showing that all the amplitudes $c_{l, m}$ in the various output channels $l, m$ are small.

[40] We also note that the intuitive fact that $\left|m_{0}(k)\right|^{2}$ is minimal in $k=k_{0}$ leads to a real value for $\beta$.

[41] Note that $\operatorname{Re} m_{0}^{\prime}$ has to be positive for consistency of the present low- $\left|m_{0}\right|$ theory: with the reasoning leading to Eq. (D1), we get the approximation for the resolvent $\left\langle\mathbf{r}\left|\mathcal{G}\left(E+i 0^{+}\right)\right| \mathbf{r}_{0}\right\rangle \simeq-A F(\mathbf{r}) F\left(\mathbf{r}_{0}\right) / m_{0}(k)$. The linearization procedure in $k$ around $k_{0}$ leads to $m_{0}(k) \simeq$ 
$\left(\beta m_{A} / \hbar^{2} k_{0}\right)\left[E-E_{0}+i \hbar \Gamma / 2\right]$, where $E=\hbar^{2} k^{2} / 2 m_{A}$, $E_{0}=\hbar^{2} k_{0}^{2} / 2 m_{A}$ and $\Gamma$ is given by Eq. (23). All the complex poles of the analytic continuation of the resolvent have a negative imaginary part, so that one should have $\Gamma>0$ and therefore $\beta>0$. This is compatible with the 'motion' of the low-imaginary part eigenvalues of $M$ in the complex plane for increasing $k$, see Fig 6

[42] M. Rusek, J. Mostowski, and A. Orlowski, Phys. Rev. A 61, 022704 (2000).

[43] One can simply use perturbation theory to understand these facts, expanding $M^{\infty}(k)$ to first order in $k$ and treating the linear terms in $k$ as a perturbation.

[44] We restrict to $s$-wave scattering for the $A$ waves, since we expect the $p$-wave scattering amplitude to vanish generically as $k^{2} a_{\text {ho }}^{3}$ at low $k$. There may be values of $a / a_{\text {ho }}$ leading to a $p$-wave resonance, but we assume that they differ from the locations of the $s$-wave resonances.

[45] R. A. Duine and H. T. C. Stoof, Phys. Rep. 396, 115 (2004).

[46] V. V. Flambaum, G. F. Gribakin, and C. Harabati, Phys. Rev. A 59, 1998 (1999).

[47] M. Olshanii, Phys. Rev. Lett. 81, 938 (1998).

[48] T. Bergeman, M. G. Moore, and M. Olshanii, Phys. Rev. Lett. 91, 163201 (2003).

[49] V. Peano, M. Thorwart, C. Mora, and R. Egger, New J. Phys. 7, 192 (2005).

[50] P. O. Fedichev, M. J. Bijlsma, and P. Zoller, Phys. Rev. Lett. 92, 080401 (2004).

[51] D. S. Petrov, M. Holzmann, and G.V. Shlyapnikov, Phys. Rev. Lett. 84, 2551 (2000).

[52] H. Moritz, T. Stöferle, K. Günter, M. Köhl, and T. Esslinger, Phys. Rev. Lett. 94, 210401 (2005).

[53] T. Stöferle, H. Moritz, K. Günter, M. Köhl, and T. Esslinger, Phys. Rev. Lett. 96, 030401 (2006).

[54] In fact, to first order in the perturbation series the term $r^{2}$ gives an energy shift proportional to $\hbar \omega\left(a / a_{\mathrm{ho}}\right)^{2}$, while the term $\mathbf{R} \cdot \mathbf{r}$ gives zero to first order, and a complex shift proportional to $\hbar \omega\left(a / a_{\mathrm{ho}}\right)^{2}$ to second order.

[55] One may wonder if one can, from this formalism suited to the case $m_{A} \ll m_{B}$, propose an approximate formula giving the value of $a^{-1}$ close to the resonance with a negative $a_{\text {res }}^{-1}$ and leading to the desired value of $a_{\text {eff }}$. We find that the answer is yes if one imposes the constraint $\left|a_{\text {ho }} / a_{\text {eff }}\right| \ll \alpha^{1 / 2}$, in which case one has the expansion

$$
a^{-1}=(1+\alpha) a_{\mathrm{eff}}^{-1}-\frac{\sqrt{2}}{a_{\mathrm{ho}}} \alpha^{3 / 2}+a_{\mathrm{ho}}^{-1} O\left(\alpha^{5 / 2}\right) .
$$

The first term in the right-hand side actually corresponds to the Born approximation. Because of the imposed constraint on $a_{\mathrm{ho}} / a_{\mathrm{eff}}$, this formula may be not very useful in practice.

[56] Some reader may fear that the matter wave $A$ spends a very long time 'inside' a given scatterer because scattering is resonant. However the Wigner time delay [57], $\tau(k)=2 m_{A} \delta^{\prime}(k) / \hbar k$, where $\delta(k)$ is the $s$-wave phase shift, has here a negative value $\tau \sim-30 \hbar / E_{r}^{A}$ (for $a_{\text {eff }}=d, k a_{\text {eff }}=1 / 3$ and using the low- $k$ approximation $\left.\delta(k) \simeq-\operatorname{atan} k a_{\text {eff }}\right)$.

[57] E.P. Wigner, Phys. Rev. 98, 145 (1955).

[58] In particular, this would allow to discriminate long dwell times due to localization effects from a parasitic matter wave trapping effect in the scattering medium, due e.g. to the formation of a weakly bound state between a matter wave atom and a trapped atom as discussed in $1 \mathrm{IB}$ Note that this particular spurious contribution to $N_{\text {rem }}(\tau)$ could also be eliminated by switching back $a_{\text {eff }}$ to a low value just before measuring $N_{\text {rem }}(\tau)$, so as to transform the weakly bound states into deeply bound states that can be discriminated from free atoms.

[59] P. Lemonde, O. Morice, E. Peik, J. Reichel, H. Perrin, W. Hänsel, C. Salomon, Europhys. Lett. 32, 555 (1995).

[60] Micro-gravity can presently be obtained e.g. at ZARM (a 120 meters high drop-tower facility in Bremen, Germany, where an experiment with ultracold atoms is planned 61]), on airplanes performing parabolic flights (experiments with atomic clocks on parabolic flights have been reported in [62]), or on an orbiting space station (experiments with ultracold atoms on the International Space Station have been planned by the European Space Agency).

[61] E. Rasel, J. Reichel, private communication.

[62] P. Laurent, P. Lemonde, E. Simon, G. Santarelli, A. Clairon, N. Dimarcq, P. Petit, C. Audoin, and C. Salomon, Eur. Phys. J. D 3, 201 (1998).

[63] A careful reader may fear that the fraction in the right hand side of Eq. [C6 is indeed of large modulus but almost real, so that its imaginary part is actually not large. As shown in the Appendix $D$ the vector $\overrightarrow{v_{0}}$ is almost real so that the numerator of the considered fraction is almost real.

[64] In the limit $r \rightarrow+\infty, F(\mathbf{r})$ involves a factor $e^{i k r} / r$ so it cannot be real for all $r$. However, using the calculation of the Appendix $[$ one finds that the angular average of $|F(\mathbf{r})|^{2}$ is of order $\operatorname{Im} m_{0}$, so that the imaginary part of $F(\mathbf{r})$ at large $r$ is $O\left(\operatorname{Im} m_{0}\right)^{1 / 2}$ and tends to zero with $m_{0}$.

[65] From Schwartz inequality $\left|\sum_{j} x_{j} \exp \left(i k \mathbf{n} \cdot \mathbf{r}_{j}\right)\right|^{2} \leq\|\vec{x}\|^{2} \times$ $N$. An accurate estimate of the maximal eigenvalue of $M_{I}$ 24] gives a value $\propto k N /(k L)^{2}$ in the large $k L$ limit, where $L$ is the diameter of the scattering medium, that is a value $\propto(k L)^{2}$ times smaller than the present upper bound.

[66] G. Bruun, Y. Castin, R. Dum, K. Burnett, Eur. Phys. J. D 7, 433-439 (1999).

[67] R. P. Feynman and A. R. Hibbs, "Quantum Mechanics and Path Integrals", Mc Graw-Hill (1965), Chap. 4.

[68] $\int_{0}^{\infty} d \rho \rho^{2} \exp \left(i x \rho^{2}\right)=\sqrt{\pi / 16 x^{3}} \exp (3 i \pi / 4)$ for $x>0$. The result for $x<0$ is the complex conjugate of the latter expression. 